\title{
POSTER SESSION 1: ATTENTION AND MEMORY, DEMENTIA, STROKE AND AGEING
}

\section{5: The Contribution of Worry, Anxiety and Thought Suppression to Performance on the Components of Working Memory in a Non-clinical Sample}

\author{
Carolyn Matthews and Simon F. Crowe
}

Psychological Science, La Trobe University, Melbourne, VIC, Australia

The aim of this study was to evaluate the contribution of worry to the pre-

diction of the components of working memory in a non-clinical sample. Sixty-one healthy adults ( 31 men and 30 women) ranging in age from 18 to 63 years were administered three questionnaires and six working memory tasks. The questionnaires were the Worry Domains Questionnaire, the State-Trait Anxiety Inventory, and the White Bear Suppression Inventory. The working memory tasks were the Digit Span task (forward and reversed), the Spatial Span task (forward and reversed), the Visual Patterns Test, and a dual performance task (digit recall plus visual tracking). Separate hierarchical regression analyses were conducted on each of the dependent measures to examine the contribution of the independent variables to the various aspects of working memory. The results indicated that worry was a significant contributor to the prediction of working memory performance. However, contrary to our hypothesis, worry did not contribute to verbal working memory or to the central executive tasks. Worry did make a significant contribution to the performance of spatial span backwards and to the dual task. Further analysis indicated that worry was negatively associated with working memory performance. Thought suppression was significantly and positively correlated with anxiety and with worry, and was also positively associated with working memory performance on the verbal, spatial, and central executive tasks. In addition, thought suppression was found to be a significant predictor of central executive performance. These issues were discussed in terms of contemporary models of anxiety and working memory functioning.

\section{1: Working Memory Ability and Card Sorting Test Performance}

\section{Clive Skilbeck and Janine Martin}

Psychology, University of Tasmania, Hobart, TAS, Australia

Drevious research involving patients with frontal lobe lesions or Pchizophrenia has indicated a possible relationship between impaired spatial working memory and poor performance on the Wisconsin Card Sorting Test (WCST), although findings have been inconsistent. The present study investigated the role of auditory, visual, and spatial working memory in card-sorting test performance. Forty-seven university students were administered computerised versions of WCST and Madrid Card Sorting Test (MCST), and four working memory tasks: Digit Span Backwards, Letter-Number Sequencing, Visual Patterns Test, and Spatial Span Backwards. Results revealed a significantly higher percentage of total errors were made on MCST than on the WCST. As there are major differences 
between the two tests; visual/no visual feedback, disclosure/non-disclosure of sorting criteria, ambiguous/unambiguous cards and error definitions, the WCST data was rescored to remove ambiguous cards and to reconcile error definitions. Further analyses of high vs. low scorers on each of the working memory tasks revealed low scorers made a significantly higher percentage of total and non-perseverative errors than high scorers, on both card-sorting tests. Although there were similar findings across all modalities a significant interaction on LNS suggests a greater role for auditory working memory in MCST performance. However, the omission of visual feedback in the MCST may account for the increased role of auditory working memory and is, therefore, the focus of ongoing research.

\section{1: Examination of Hemispheric Contributions to Switching Abilities: A Replication with a Different Paradigm}

Yana Suchy and April E. Gold

Psychology, University of Utah, Salt Lake City, UT, USA

Drior research examining hemispheric contributions to switching has been inconsistent, with some studies suggesting no hemispheric dominance (Demakis, 2003, Dove et al., 2000), others suggesting left-hemisphere dominance (e.g., Rogers et al., 1998), and others yet suggesting right-hemisphere dominance for switching (Volz et al.,1997). These inconsistencies could be explained by the fact that successful switching may rely on both inhibition and initiation, which themselves appear to be differentially subserved by the right and the left hemispheres, respectively (e.g., Stuss et al. 2002). In a recent study (Suchy et al., 2003), we examined the relative involvement of initiation and inhibition in switching, as well as the relative roles of the two hemispheres in these processes. We used a modification of a switching task that allowed examination of switching between $(\mathrm{B} / \mathrm{W})$ and within $(\mathrm{W} / \mathrm{N})$ hemispheres. We found that switching from right (inhibition) to left (initiation) hemisphere is faster than switching in the opposite direction, and that switching $\mathrm{B} / \mathrm{W}$ (placing greater demands on initiation) is faster than switching W/N (placing greater demands on inhibition). These results support the notion that initiation and inhibition (and the respective hemispheres) both contribute to switching, and suggest that inhibition may play a more prominent role than initiation. Because this was the first study of its kind, we set out to replicate the results with different stimuli and a substantially different switching task design. We again found faster switching for $\mathrm{B} / \mathrm{W}$, as compared to $\mathrm{W} / \mathrm{N}, t(40)$ $=3.75, p=.001$, Cohen's $d=.59$; and for right-to-left, as compared to left-toright, $t(40)=4.58, p<.001$, Cohen's $d=.72$. Although the present results suggest that these effects are stable, further research is needed to test alternative explanations of these findings.

\section{1: Memory Correlates of Rhinal Cortex and Hippocampal Volumes in Patients with Mesial Temporal Sclerosis}

Catherine E. Meade ${ }^{1,2}$, Stephen C. Bowden ${ }^{2,1}$, Fiona J. Bardenhagen ${ }^{1}$ and Mark J. Cook ${ }^{1}$

1. Clinical Neurosciences, St Vincent's Hospital, Melbourne, VIC, Australia

2. School of Behavioural Science, The University of Melbourne, Melbourne, VIC, Australia

The function of primate rhinal cortex, comprising the entorhinal (ErC) and perirhinal (PrC) cortices, has been extensively studied. Translating 
animal models to human memory has been limited by the technological problems associated with characterising neural structures in vivo. Neuropsychological correlates of hippocampal and rhinal cortex volume changes were examined in a sample of 61 temporal lobe epilepsy patients with mesial temporal sclerosis (33 left, 28 right). Patients were administered the Wechsler Adult Intelligence Scale (Revised or Third Edition), Wechsler Memory Scale (Revised or Third Edition) and a spatial maze task. Multiple regression analysis was used to examine neuropsychological data, together with rhinal cortex and hippocampal volumes, collected in our earlier study (unpublished observations, OBrien et al.). The only significant predictor of verbal memory function was the difference score between the volume of left hippocampus and the left PrC. Spatial maze scores were predicted by the bilateral sum of $\mathrm{ErC}$ volume. The difference score between the left hippocampus and left $\operatorname{PrC}$ volumes was the most powerful predictor of verbal episodic memory. Right hippocampal volume was not a significant predictor of nonverbal episodic memory. Verbal and nonverbal semantic memory were not significantly predicted by any combination of rhinal cortex structures. Results suggest lateralised memory function for the hippocampus and $\mathrm{PrC}$, in contrast to the bilateral role of the ErC. The hippocampus and $\operatorname{PrC}$ may act on memory function through an opposing relationship. A differentiation between hippocampal and subhippocampal components in terms of episodic and semantic memory, respectively, was not supported by the current data.

\title{
72670: Development and Validation of a Computerised Visual Memory and Learning Test
}

\author{
David Shum ${ }^{1,2}$, John O'Gorman ${ }^{1,2}$, Joanne Oram³ ${ }^{3}$, Dale Fogarty ${ }^{4}$, Helen Tinson ${ }^{3}$ \\ and Kerryn Neulinger ${ }^{1,2}$ \\ 1. Applied Cognitive Neuroscience Research Centre, Griffith University, Brisbane, QLD, Australia \\ 2. Applied Psychology, Griffith University, Brisbane, QLD, Australia \\ 3. Department of Neurosciences, Princess Alexandra Hospital, Brisbane, QLD, Australia \\ 4. Brain Injury Rehabilitation Unit, Princess Alexandra Hospital, Brisbane, QLD, Australia
}

Visual memory is defined as the ability to recall or recognise visual patterns that do not lend themselves easily to verbal encoding (Milner, 1971). Impairment in visual memory is a commonly observed symptom in many neurological conditions and it has been found to be a predictor of functional and vocational outcomes in individuals with brain injury. The development of tests of visual memory and learning has lagged behind that of its verbal counterpart. This is because it is difficult to construct visual stimuli that are unfamiliar, complex and difficult to verbalise (Heilbronner, 1992). Eadie and Shum (1995) proposed that Chinese characters meet these criteria (for those who have not learned this language) and showed that the Shum Visual Learning Test (Shum, O'Gorman, \& Eadie, 1999), which uses a set of relatively complex Chinese characters with low verbalisation index, is sensitive to lateralised brain damage. Recently, a computerised version of this test was developed to increase its clinical utility. This study aims to describe this latest version of the Shum Visual Learning Test. The study will present data to compare the equivalence of the computerised to the original version of the test and evaluate its reliability. Performance of patients with localised damage to the left and right temporal lobes will also be used to support the validity of this test. 


\section{3: Cognitive Profile of vCIND Patients and Progression in Dementia}

S.Y. Tay', D. Yeo ${ }^{1}$ and C.P.L.H. Chen ${ }^{2}$

1. Neurology, Singapore General Hospital, Singapore, Singapore

2. Neurology, National Neuroscience Institute, Singapore, Singapore

Background: The concept of Mild Cognitive Impairment (MCI) has been widely used in defining the stage between normal ageing and dementia that is associated with an increased risk of developing dementia. Although memory impairment with preserved functional abilities has been the main classification criteria, recent research has explored the heterogeneity of this concept and examined the role of non-memory cognitive impairment. Studies that subclassify MCI patients according to their cognitive profile suggest that whilst amnestic-MCI patients are at an increased risk of developing dementia (Busse et. al., 2003), this risk is increased in patients with cognitive impairment beyond memory loss (Bozoki et. al., 2001). The applicability of these findings to vascular Cognitive Impairment Not Dementia (vCIND) has not been explored extensively.

Aim: To investigate the incidence of dementia associated with different vCIND subgroups.

Methods: Patients with ischaemic stroke were cognitively assessed in the domains of attention, language, verbal memory, visual memory, visuoconstruction and visuomotor speed within 6 months of their index stroke and 1 year later. Dementia was diagnosed using DSM-IV criteria. Patients who did not meet DSM-IV criteria, but were impaired in one or more cognitive domains, were classified as vCINDmem+ (multiple cognitive impairments including memory), vCINDmem (only memory impairment) and vCINDmem- (impairment in non-memory domains).

Results: 69 patients diagnosed with vCIND at baseline were included in this study, of which $43.5 \%$ were vCINDmem+, $23.2 \%$ were vCINDmem and $33.3 \%$ were vCINDmem-. At 1-year follow-up, $16.7 \%$ of the vCINDmem+ patients compared to $4.3 \%$ of the vCINDmem- patients developed dementia. None in the vCINDmem group declined.

Conclusion: Over a 1-year period, the risk of developing dementia was highest in the vCINDmem+ group compared to other vCIND subgroups $(\mathrm{OR}=7.6$; 95\% CI 0.8-69.0).

\section{7: Long-Term Cognitive Treatment In Alzheimer Disease: A Single-case Study}

Alberto L. Fernandez and Laura M. Manoiloff

Neuropsychology, Institute of Neurosciences, Cordoba, Argentina

The effects of long-term treatment in a demented patient were evaluated in this study. One individual diagnosed with Alzheimer dementia was treated with neuropsychological rehabilitation techniques as well as drugs for a period of two years and ten months. An A-B-A-B design was performed for the cognitive treatment. Neuropsychological treatment consisted of a combination of direct re-retraining and training in activities of daily living. Cognitive performance was monitored with the Mattis Dementia Rating Scale. Results showed improvement and a slower decline during the treatment phases (A) as compared to the non-treatment phases (B). Conceptualisation, and attention were the subscales that benefited the most followed by the 
memory subscale. Long-term treatment showed effectiveness in AD treatment. Although cognitive drugs may have been beneficial, neuropsychological rehabilitation played the most important role in the success of this treatment, appearing as a necessary condition.

\title{
71971: Utility of the Clock Drawing Test in Differentiating Dementia and Traumatic Brain Injury in the Elderly
}

Tony J. Kreuch

Healthsouth Hospital, Albuquerque, NM, USA

This study examined the utility of the clock-drawing test in differentiating between elderly patients with progressive dementia and those with traumatic brain injury. It was hypothesised that elderly patients with known progressive dementias would perform more poorly than those with acute traumatic brain injury or elderly controls. Subjects were 30 rehabilitation patients, over age 65. The following groups were compared: (1) Dementia Group (elderly patients with diagnosed progressive dementias); (2) Traumatic Brain Injury Group (criteria: positive LOC, positive neuroradiological findings, and ER GCS of 12 or lower); (3) Orthopedic Controls. No significant difference between groups was found for age or educational level. The clock-drawing test, using standardised instructions and scoring was administered. Dependent measures included total raw score, number placement score, hand placement score and visuospatial performance. A one-way ANOVA found a significant between-group effect for raw scores and all sub-scores $(p<.01)$. Post-hoc multiple comparisons analyses yielded significant mean differences between the dementia group and the TBI Group on all dependent measures (Progressive Dementia Group significantly lower). The progressive group was also significantly lower than the orthopedic controls $(p<.01)$, however, the orthopedic group and the TBI group scores were not significantly different. Results indicate that the clockdrawing test is useful in differentiating between elderly patients with acute traumatic brain injury and those with progressive dementia (e.g., Alzheimer's disease). The orthopedic group, used as a control group in the study, may not be representative of a normal elderly sample (given the setting), and additional research using a normal ageing group may be useful regarding test utility for differentiating cognitive impairment in elderly TBI patients from normal elderly.

\section{4: Evaluation of a Virtual Reality-Based Memory Training Program for Persons of Early-Stage Dementia of Alzheimer Type: A Preliminary Study}

\author{
David W. Man, Sing Fai Tam and Christina W.Y. Hui-Chan \\ Rehabilitation Sciences, The Hong Kong Polytechnic University, Hong Kong, Hong Kong
}

Background: Dementia is a major cause of disability and accounts for a considerable proportion of health care expenditure. Memory is usually the first cognitive function to be affected by the onset of dementia of the Alzheimer's type (DAT). Evidence of cognitive rehabilitation of memory in early stage DAT suggests that learning is possible in people with DAT and they are capable of learning and retaining information. The present study thus developed and evaluated a non-immersive virtual reality (VR)-based memory training programme, in which behavioural responses within such environments can be recorded and measured. 
Method: A control group pre- and post-test quasi-experimental design was applied to 45 DAT subjects who were randomly assigned into three groups respectively: VR group, non-VR group, and a control group. Summative outcome measures included the Chinese Version of Mattis Dementia Rating Scale, Everyday Memory Questionnaire, and rating scales to assess knowledge, skills and self efficacy of subjects in performing community living activities. During a 4-week study period, the three groups underwent a 10session, two-level program in training memory skills.

Initial results: ANOVA and MANOVA showed that both the VR and NonVR programs were effective in improving memory skills and self efficacy in community living skills; and were significant better than control group. Moreover, subjects would be stratified according to education, gender, site and duration of injury, by means of ANCOVA, to control confounding variables and/or study the possible interaction effects of these factors.

Conclusion: VR approaches are initially found to be effective and efficacious in memory training for DAT.

\section{5: Semantic Verbal Fluency in Alzheimer's Disease: Approaches Beyond Traditional Scoring System}

Evrim March

Department of Psychology, University of Melbourne, Melbourne, VIC, Australia

Semantic and phonemic verbal fluency testing forms an integral part of neuropsychological assessment. This paper aims to discuss possible approaches to semantic verbal fluency testing beyond that provided by the traditional scoring system (i.e., total number of correct words). These approaches are examined through use of semantic verbal fluency data from 26 probable mild Alzheimer's disease patients (MMSE mean $=20.5)$ and 26 older adults $($ MMSE mean $=27.7)$, matched for age $($ mean $=78.8)$, education (mean $=8$ years) and gender. The paper first discusses the usefulness of recently proposed process-oriented approaches, that is, clustering (i.e., generating words within semantic subcategories) and spontaneous switching (i.e., shifting between semantic subcategories) strategies in Animal and Supermarket categories (Troyer, Moscovitch and Winocur, 1997). It is demonstrated that, in contrast with extant research findings, the variables do not distinguish the two groups particularly well. Second, the paper explores the potential advantage of two other measures, number of clusters (i.e., number of subcategories accessed) and number of repetitions (i.e., repetition of words already generated) as qualitative markers of semantic verbal fluency. Finally, drawing on to Newcombe's (1969) research, the paper argues that, rather than exploring spontaneous switching fluency ability, substantial insights into semantic verbal fluency would be gained through directly testing purposeful switching ability (i.e., alternating between given semantic categories such as Person name/Fruit), with evidence from research data. The discussion focuses on theoretical and clinical implications of these measures, with a view to expanding traditional approaches to semantic verbal fluency assessment. 


\title{
73184: Degree of Discrepancy Between Self- and Other-reported Everyday Functioning by Cognitive Status
}

\author{
Sarah Tomaszewski Farias and Dan Mungas \\ Neurology, University of California, Davis, Sacramento, CA, USA
}

\begin{abstract}
Drevious studies have shown that patients with dementia tend to overestimate their cognitive and functional abilities as compared to the reports of their caregivers. Recently, there has been interest in the preclinical stage of dementia, referred to as mild cognitive impairment (MCI). Past research has not examined whether this clinical group also tends to underestimate their deficits. In this study we examined whether degree of discrepancy between patient and informant-reported everyday function was associated with cognitive status. The sample consisted of 111 community-dwelling older adults (45 Caucasian, 65 Hispanic, 1 Asian), and was divided into three subgroups dependent on cognitive status: cognitively normal, MCI, and demented. A difference score was calculated by subtracting the patientreported score on the Daily Function Questionnaire from the informantreported score. A higher score indicated that the informant was reporting more impairment. The difference scores for Normal and MCI groups clustered around 0, but the average difference for the Demented group was .55, indicating that the Demented group was underestimating their level of functional impairment. Furthermore, the difference scores for the Demented group significantly differed from both those of the Normal and MCI groups, while the difference scores for the Normal and MCI groups did not significantly differ. These results provide preliminary evidence to suggest that self-reported functional abilities may yield valid and reliable information in older adults falling in the MCI category.
\end{abstract}

\section{5: Neuropsychological Diagnosis and Qualification of Dementia}

Janna M. Glozman

Psychology, Moscow State University, Moscow, Russian Federation

The paper proposes the critical analysis of Russian and American diagnostic criteria for dementia. Neurological and psychiatric aspects of the problem and some reasons for misdiagnosis of dementia are discussed. The main cause for the overdiagnosis of dementia is an imprecise current definition of dementia. The obligate and logical criteria of dementia are proposed. Some approaches and methods to a neuropsychological diagnosis of dementia (available for medical doctors) are analysed. The results of a combined psychometric and qualitative analysis of cognitive and executive disturbances (Luria's battery) in 3 age matched groups of elderly patients with common pathology: vascular encephalopathy, vascular dementia and Alzheimer's disease are presented. A combined approach such as this permitted us: (1) to identify differential symptoms in the executive behaviour of different groups of patients; (2) to determine specific patterns of cognitive disturbances for different kinds of executive disturbances; (3) to demonstrate that human executive behaviour is conditioned by the close integration and interaction of the three functional units of the brain postulated by Luria (the activational unit, the unit for information processing and storage, and the unit for programming, regulation, and monitoring); and (4) to reveal the role of dysfunction in different cortical and subcortical brain regions for the genesis of these cognitive and executive disturbances in each 
type of dementia. An evolution and progression of cognitive disturbances up to the appearance of vascular dementia is predominantly due to regulatory and operational disorders connected to cortical brain regions. A "corticalisation" of the cognitive and executive disturbances takes place. The evolution of Alzheimer's disease is realised via the consecutive "frontalisation" and "subcorticalisation" of disturbances, that is, by superimposed neurodynamic and regulatory impairments upon operational ones.

\section{2: Early Assessment of Dementia: The Contribution of Different Memory Components}

Pauline E. Spaan ${ }^{1}$, Jeroen G. Raaijmakers ${ }^{1}$ and Cees Jonker ${ }^{2,3}$

1. Psychonomics, University of Amsterdam, Amsterdam, Netherlands

2. Institute for Research in Extramural Medicine, Vrije Universiteit, Amsterdam, Netherlands

3. Psychiatry, Vrije Universiteit, Amsterdam, Netherlands

The present research investigated whether the differentiation between various memory components (i.e., episodic, semantic, implicit, working memory) contributed to the early assessment of dementia. Whereas clinical neuropsychological assessment primarily employs tests of episodic memory functioning, the present research used a computerised test battery reflecting the different memory components mentioned above. In this way, it was determined which combination of measures (i.e., memory components) was most accurate in predicting dementia, before the diagnosis could officially be made. This memory test battery was administered to a heterogeneous sample (concerning their global cognitive status measured by the MMSE) of initially non-demented elderly subjects $(n=147)$. These subjects were all community dwelling elderly persons, derived from the population-based "Longitudinal Aging Study Amsterdam" (LASA). In order to examine the profile of preclinical dementia, subjects were tested twice: at baseline (T1) all subjects were non-demented according to DSM-IV criteria, while 2 years later (T2) a subgroup had developed dementia. Dementia was best predicted, two years before diagnosis, by the combination of a paired-associate learning test and a priming measure derived from a perceptual identification task. Thus, performance of the preclinically demented subjects was best characterised, relative to cognitively impaired but non-demented subjects, by an inability to benefit at recall from semantic relations and by absent repetition priming effects. In contrast, a purely episodic memory measure (a ten word list-learning test which demanded free recall of semantically unrelated words) only showed decline relative to controls once dementia was officially diagnosed (at T2). It is concluded that in addition to testing episodic memory functioning, it is particularly important to be aware of semantic and implicit memory deficits in the early assessment of dementia.

\section{4: Mechanism of Closing-in Phenomenon on Figure-copying Tasks in Alzheimer's Disease}

Byung H. Lee, Juhee Chin, Sue J. Kang, Eun-Joo Kim, Key C. Park and Duk L. Na Department of Neurology, Samsung Medical Centre, Sungkyunkwan University School of Medicine, Seoul, South Korea

The "closing-in" phenomenon is defined as a tendency to copy near or reported that the closing-in phenomenon is more closely associated with 
diffuse rather than focal brain lesion and it can be regarded as one of the specific neuropsychological markers of Alzheimer's disease (AD). However, the mechanisms underlying the closing-in phenomenon have not been fully elucidated. We posit that closing-in may be related to the patients' compensatory strategies for visuospatial dysfunction or visuospatial working memory deficit. Thus it is expected that as the complexity of the target figure is increased or the distance from the target to the copying space is increased, the closing-in phenomenon will increase. We recruited $15 \mathrm{AD}$ patients who showed the closing-in on a screening test and 15 age, sex and education matched normal controls. Each subject performed 12 copying trials (four different stimuli $\mathrm{x}$ three different distances) in random order. The proximity (Y-axis) of the subject's drawing (X-axis) toward the target was plotted as a function of distance from the left end to the right end of the drawing and the degree of closing-in was computed by the slope of the regression line. The slope of AD patients' drawings differed as the figure complexity ( $p=.003$ ) but the effect of distance from the target to the starting point was not found. There was a linear relationship between the complexity of figures and the magnitude of closing-in $(p=.004)$, indicating that the severity of closing-in increased in proportion to the complexity of figures. Our results are most consistent with the visuospatial working memory hypothesis. That is, copying near the target figure may be a strategy to compensate for patients' visuospatial dysfunctions or visuospatial working memory deficits.

\section{1: Inhibition of Prepotent Responses in Parkinson's Disease}

Allison M. Fox ${ }^{1,2}$, Elizabeth J. Morton ${ }^{1}$, Jonson Moyle ${ }^{1}$ and Jeffrey M. Rogers ${ }^{1}$

1. Psychology M304, The University of Western Australia, Perth, WA, Australia

2. Private Practice, Perth, WA, Australia

Q vent-related potentials (ERPs) provide a non-invasive technique for Cexamining the nature of processing required during task performance, and are particularly useful when overt behavioural measures cannot be obtained. In addition, they can provide a sensitive indicator of injury in various clinical disorders when behavioural consequences may not be observable in overt behaviour. The present study examined response inhibition processes by recording ERPs whilst participants performed a Go-Nogo task that required responding to certain letters presented on a computer monitor and withholding responses to other letters. Five participants with mild Parkinson's disease (PD) and nine control participants completed the task. Results from the behavioural analyses indicated that the PD group responded more slowly than controls. Results from the analyses of stimulus-locked ERP components indicated that there was a significant reduction in the amplitude of the Nogo-N2 component in the PD group, suggesting impairment in aspects of response inhibition. Previous studies using highdensity electrode mapping techniques have identified sources for this component in the right orbito-frontal cortex and anterior cingulate cortex, suggesting the involvement of these regions in PD relatively early in the course of the disease. Implications of these findings for the management of clients with PD will be discussed. 


\title{
73492: Prevalence of Micrographia on Free Writing Versus Copying Task in Idiopathic Parkinson's Disease
}

Eun-Joo Kim, Key Chung Park, Byung Hwa Lee, Won Yong Lee and Duk L. Na Department of Neurology, Samsung Medical Centre, Seoul, South Korea

\begin{abstract}
$\mathrm{M}$ icrographia is a characteristic sign of idiopathic Parkinson' disease (IPD). Micrographia usually manifests itself in two forms: Consistent Micrographia (CM) and Progressive Micrographia (PM). Prior studies that investigated the prevalence of $\mathrm{CM}$ reported it to be about $10-15 \%$. However, the definition of micrographia in those studies was ambiguous. This study investigated the prevalence of $\mathrm{CM}$ and $\mathrm{PM}$ on the basis of letter and figure copying tasks that were performed in both IPD patients and normal controls. Seventy five IPD patients (31 men and 44 women with mean age of 64.6 59.4 ) and 30 sex/age matched controls received tasks consisting of two parts: free writing and copying task. In the free writing task, the subjects were asked to write the names of days of the week in Korean, repeating it three times in their own writing size. In the copying task which consisted of four conditions (letter and Luria figure $\mathrm{X}$ of two different sizes), subjects were required to write or draw using a visual guide of two sample letters or figures. CM was defined by the average size of the free writings and the letter or figure copyings of the patients that was 2SD below the mean of the controls. PM was defined by the slope (beta value) of the regression line for patient's free writing or copying tasks that was $2 \mathrm{SD}$ below the meanof the controls. In free writing, we found only one out of 75 patients who met the CM definition and only two patients fulfilling the definition of PM. In contrast, copying tasks revealed that in the letter task the prevalence of CM and PM was $31 \%(23 / 75)$ and $12 \%$ (9/75) respectively, and in the Luria figure copying tasks it was $24 \%(18 / 75)$ and $16 \%(12 / 75)$. Our study suggests that the prevalence of micrographia is much higher than it had been previously thought, and that the definition of microgrphia based on the copying task would be more reasonable than the one based on free writing.
\end{abstract}

\section{4: Focal Basal Ganglia Damage and Procedural Memory}

Sara Cavaco ${ }^{1}$, Steven W. Anderson ${ }^{2}$, Alexandre Castro-Caldas ${ }^{1}$ and Hanna Damasio ${ }^{2}$

1. Faculdade de Medicina da Universidade de Lisboa, Lisboa, Portugal

2. University of lowa College of Medicine, lowa City, IA, USA

There is evidence that damage to the basal ganglia impairs acquisition of perceptual-motor skills. The data have come primarily from studies of patients with neurodegenerative diseases (Parkinson's and Huntington's disease), and have relied on a limited number of laboratory tasks (i.e., Serial Reaction Time Test, Rotor Pursuit, Mirror Tracing). The goal of this study was to further explore the impairments of procedural memory that result from basal ganglia dysfunction, by examining the performances of subjects with focal lesions in this region on a new set of procedural memory tasks. The performances of 8 subjects with unilateral basal ganglia vascular lesions ( 2 left; 6 right) were compared to 25 normal control subjects on 5 new laboratorycontrolled tasks based on real world activities, encompassing a range of perceptual-motor requirements. The tasks were administered in two time periods (Time 1 and Time $2-24$ hours). The first task consisted of pressing a sequence of 5 buttons without visual guidance. The second task consisted of manually tracking constant series of 8 target locations using a joystick. The 
third task consisted of weaving fabric with an actual loom, by performing a recurrent 5-step routine. The fourth consisted of tracing moving geometric figures with a stylus on a touch screen monitor. The fifth consisted of pouring water from a small watering can into graduated containers, from a point at $20 \mathrm{~cm}$ distance. The basal ganglia group showed a trend toward reduced acquisition and/or retention on 4 of the 5 tasks (all except the 2 nd task), relative to the control group, but the differences between groups were statistically nonsignificant. Each basal ganglia subject showed impaired acquisition and/or retention on at least one of the tasks. The findings provide further evidence for impairment of perceptual-motor skill learning and retention following basal ganglia damage, and suggest that lesion and patient characteristics may influence expression of the impairment.

\section{9: Neuropsychological Dysfunction and Thalamic Stroke Lesions}

Luis L. Gutierrez Cabello

Josep Trueta Hospital of Girona, Service of Neurology, and University of Barcelona, Spain

Thalamic stroke lesions are associated with cognitive and behavioural
impairments.

Aim: To examine the relationship between thalamic stroke lesions and cognitive dysfunction.

Patients and Method: 15 people with stroke thalamic lesions were studied (mean age was 55.8 SD: 16.8 years; $66 \%$ were male; and the education level was $7 S D: 3.7)$ and compared with a control group of 20 healthy subjects (mean age 67 SD: 7.6; male 50\%; and education level of 7.6 SD: 2.9). CAT and MRI examinations were performed during the first 48 hours. $31.4 \%$ of patients $(n=11)$ had an ischaemic infarction and $11.4 \%(n=4)$ had a haemorrhagic infarctions. $46 \%(\mathrm{n}=7)$ had lesions of the right hemisphere, $40 \%$ $(n=6)$ had lesions of the left hemisphere, and 13\% $(n=2)$ had bilateral lesions. A battery of neuropsychological assessments were given to measure memory, language, attention and executive functions. The affective scales used were Hamilton Rating Scale Depression (HRS-D) and Beck-Rafaelsen Mania Scale (BRMS).

Results: Significant statistical differences were found between patients with thalamic stroke lesions and the control group in the different neuropsychological tests: memory learning (RAVLT) $p<.000$; memory test recall (RAVLT) $p<.002$ and phonetic fluency $(\mathrm{PhF}) p<.010$. No statistically significant differences were observed in the locus of lesions. Comparing patients with ischaemic infarcts with those with haemorrhagic lesions, it was found the patients with haemorrhagic lesions had lower TMT-(A/B) scores $(p=.061$ and $p=.063)$; and semantic fluency, $(p=.018)$. Results from the Hamilton Depression Scale (HRS-D) indicated that patients with haemorrhagic stroke lesions had more depressive symptoms than those with ischaemic infarctions, $p=.022$.

Conclusions: Patients with thalamic stroke lesions were found to have memory and learning and executive function impairments. Lesion location (left-right) was not observed as resulting in changes in the cognitive functions. Haemorrhagic infarcts resulted in attention deficits, executive function impairments. 


\title{
73857: Rapid Motor Learning in Hemiplegia During Uninstructed Practice: A Time-series Study with Implications for Clinical Neurorehabilitation
}

\author{
Emilie F. Tijs and Thomas A. Matyas \\ School of Psychological Science, La Trobe University, Melbourne, VIC, Australia
}

\begin{abstract}
J iterature on hemiplegia rehabilitation has recently experienced a conLtroversial shift in focus. It has been suggested that specific techniques administered by allied health professionals are not as important as sheer quantity of practice, thus calling into question the value of specialist interventions. This meta-analysis of longitudinal single-case studies investigates the effects of uninstructed practice alone.

Nine participants with hemiplegia attended between 10 and 30 daily training sessions, during which they repetitively practiced three upper limb tasks without any specific intervention. The tasks were copying a circle, a line, and a triangle. Pen trajectories were recorded on a digitising pad, and kinematic data was standardised against an age-matched normative sample. Temporal variables (movement speed, duration, and jerkiness) were often initially grossly abnormal, and improved dramatically with practice. Spatial variables (roundness of circles, horizontality of lines, etc) were comparatively well preserved initially. With practice, spatial performance was either maintained, or improved slightly. Results suggest a tendency to prioritise the functional aspects of the task (spatial accuracy) at the expense of temporal performance. The striking improvements in temporal performance without loss of spatial accuracy suggested that substantial gains in coordination occurred with practice. Those with greater initial deficit tended to show faster improvement, suggesting that motor impairment facilitates practice-dependent learning. The tilt of the pen was also measured to indicate normality of arm position. Pen tilt was consistently in the abnormal range, and did not improve with practice. Results suggest that although coordination may improve with task-specific practice, postural abnormalities are likely to persist without specific intervention.
\end{abstract}

\section{0: The Robustness of the Spot-the-Word Test after Stroke}

David C. Gillespie', Audrey Bowen ${ }^{1}$ and Jonathan K. Foster ${ }^{2,3}$

1. Human Communication and Deafness Group, University of Manchester, Manchester, United Kingdom

2. Medical School, University of Western Australia, Crawley, WA, Australia

3. Neuroscience Unit, Health Department of Western Australia, Mt Claremont, WA, Australia

$\mathrm{C}$ Tomparing current with estimated premorbid performance helps identify acquired deficits and assists rehabilitation planning. Tests of reading pronunciation are often inappropriate for stroke patients who may have articulatory problems. Lexical decision tasks offer an alternative approach. The Spot-the-Word test (STWT; Baddeley et al., 1993) requires identification of the real word in 60 word/non-word pairs. Although the test is believed to be resistant to the effects of cerebral damage, neither its validity nor reliability has been demonstrated following stroke. We recruited a consecutive sample of stroke patients $(n=56)$ and administered the STWT at four and 16 weeks post-stroke. For each patient, a control subject, matched for age and initial STWT scaled score, was recruited. Controls were also readministered the STWT. Statistical analysis was by limits of agreement (LOA; Bland and Altman, 1986) between four and 16-week STWT scaled scores. LOA were found to be larger for patients (-3.6 to 3.9) than for con- 
trols ( -2.3 to 2.7$)$. Eight patients (14\%) obtained scale score improvements at 16 weeks greater than the upper agreement limit of controls. The $95 \%$ confidence interval around the $14 \%$ finding was 5 to $23 \%$. The number of patients achieving an improved score is clearly greater than the $2.5 \%$ expected by chance. This suggests that, at four weeks post-stroke, the STWT is not resistant to cerebral damage. In contrast, only one patient $(2 \%)$ deteriorated (i.e., obtained a scaled score decline more extreme than the lower agreement limit). We caution that the STWT may therefore significantly underestimate the actual premorbid level of ability in stroke patients. More work is needed to identify whether there are particular types of patients for whom the test is less robust.

\section{6: An Exploration of Multi-Modal Emotion Perception and Ageing}

Sophia J. Durrani ${ }^{1}$, Anthony P. Atkinson ${ }^{2}$, Reiner Sprengelmeyer ${ }^{1}$ and David I. Perrett ${ }^{1}$

1. School of Psychology, University of St Andrews, St Andrews, United Kingdom

2. Department of Psychology, University of Durham, Durham, United Kingdom

Gotion can be conveyed in many ways, which include facial and vocal Cexpressions of emotion and changes in body movements and postures. Current thinking suggests that specific emotions, regardless of their modality, are processed by partially separable neural mechanisms. Calder and colleagues (2003) reported a progressive decline with age in the perception accuracy of fearful and angry facial expressions. We investigated emotion perception from vocal, static facial, and dynamic gesture displays amongst adults of all ages (range 20-80 years). Results show no differences between older people and their younger counterparts in facial emotion perception. Impairments in perceiving specific emotions in some tasks were revealed that were not replicable across other tasks or modalities. This lack of convergence in our results raises questions concerning the methods used to explore dissociable emotion perception mechanisms and their interpretation. The way these results may influence our understanding of the differential effects of ageing is considered.

\section{4: Target to Target Interval (TTI) as a Determining Factor in the P300 Component in Elderly Subjects}

\section{Christopher R. Gabriel}

The Brain Centre, Singapore General Hospital, Singapore, Singapore

I n event-related potential studies, probability effects, inter-stimulus intervals (ISI) and sequence effects are usually manipulated in determining the P300 component. Literature research by Gonsalvez (1999) has determined that these manipulations can also be attributed to the temporal relationship between targets. The experimental design usually utilised is the 2-tone target and non-target oddball paradigm. However, if the target-to-target interval (TTI) is the determining factor of the profile of the P300 component, then sequence effects, ISI and probability effects can be subsumed under this experimental manipulation. A new experiment was designed where two experimental conditions were employed, a two-tone condition and a one-tone condition. The 2-tone condition followed the standard oddball paradigm with target stimuli being separated by non-target stimuli varying from none (zero non-targets) to 9 non-target stimuli. With the ISI kept constant at 1 -second, TTI ranged from $1 \mathrm{~s}$ to $10 \mathrm{~s}$. In the 1-tone condition, 
only target stimuli were presented and the intervals ranged from $1 \mathrm{~s}$ to $10 \mathrm{~s}$, counter-balancing the 2-tone experimental condition. Elderly subjects, ranging in age from 50 to 75 years with no history of neurological conditions participated in the study. Using the 10-20 system, brain electrical activity was recorded from midline frontal, central and parietal sites. Results indicated that in these subjects, P300 amplitude increased with increasing TTI with the greatest amplitude at Pz. P300 latency decreased with increasing TTI but with a difference between conditions. These findings suggest that the TTI hypothesis is a valid hypothesis. Research is currently on-going examining a clinical sample population.

\section{POSTER SESSION 2: METHODOLOGICAL AND CONCEPTUAL ISSUES AND TESTS \\ 73454: EMDR (Eye Movement Desensitisation and Reprocessing) and REM Sleep}

Masakazu Sugawara and Koich Suzuki

Psychology, Iwate University, Morioka, Japan

Background: Eye movement desensitisation and reprocessing (EMDR) is a new innovative treatment with a high success rate for psychological disturbances rooted in traumatic memory. However, the neurophysiological mechanisms of EMDR have not yet been elucidated. Why is saccadic eye movement effective for the reprocessing of previously established conditioned reflex?

Aims: The present research analysed (i)the topographical changes of EEG (and ERP) and REM sleep after EMDR treatment, and (ii) the subjective units of emotional distress (SUDs) and VOC.

Methods: Subjects (13 males and 20 females) were assigned to three groups according to varied conditions (control, provocational, and EMDR), and engaged in sets of horizontal saccadic-eye movements lasting approximately 30 seconds per set. Topographical changes of EEG and ERP activities were recorded from 14 placements over frontal, central, parietal, temporal and occipital scalp locations in the international 10-20 system with linked mastoides (A1-A2). Electrodes were also placed on the lateral canthus and above the supercillium of the left eye in order to measure the electrooculographic and electromyographic responses.

Results and Conclusions: The statistical significance of topographical EEG differences and REM densities during the pre-/post EMDR treatment situations, were evaluated using an ANONA and Mann-Whitney U test. The neurophysiological and psychological data indicate that the density of eye movement during REM sleep increased after provocation and EMDR, and the left frontal activities might indicate a treatment efficacy. It supports the hypothesis is that REM sleep is intimately involved with the mechanisms of emotional and memory reprocessing. 


\section{2: Analysis of Patient and Proxy Ratings on the Dysexecutive Questionnaire}

Raymond C. Chan ${ }^{1,2}$ and Rita Bode ${ }^{4,3}$

1. Psychology, Sun Yat-Sen University, Guangzhou, China

2. Psychiatry, The University of Hong Kong, Hong Kong, Hong Kong

3. Department of Physical Medicine \& Rehabilitation, Northwestern University, Chicago, IL, USA

4. Center for Rehabilitation Outcomes Research, Rehabilitation Institute of Chicago, Chicago, IL, USA

This study had two objectives: (1) to examine differences in the ratings of 1 patients and proxies on a measure of the dysexecutive syndrome; (2) to further explore the insight impairment problem in patients with traumatic brain injury using Rasch Analysis. A total of 179 patients (self- and proxy ratings) were obtained. Eligible proxies were significant others of the patients who lived with the patients for at least the past year. The Dysexecutive Questionnaire (DEX) is a 20-item instrument measuring cognitive, behavioural and emotional factors, rated on a 5-point frequency scale from never to very often. Rasch analysis of the ratings by the patient, a proxy, and the difference in ratings between the two describe the psychometric characteristics of the DEX. Rasch calibration of the separate patient and proxy data showed slightly more internally consistent ratings for proxies (.91) than patients (.89) while the difference between ratings was slightly less reliable (.85). At the item level, the ratings for restlessness misfit the model for both patients and proxies, and five items exhibited differential item functioning: distractibility, temporal sequencing problems, poor decision-making, knowing/doing dissociation, and lack of concern, reflecting differences in the perspectives of patients and proxies. A conversion table from patient to proxy scores shows slight differences in comparable scores along the scale. The present study confirms the findings that proxy estimates are slightly more reliable than patient estimates. The provision of a conversion table from patient to proxy scores further facilitates the interpretation of the discrepancy between the two sets of ratings.

\section{0: Factor Analysis of Neuropsychological Function on a Normal Population}

Takeuchi Al', Mie Matsui ${ }^{2}$, Kanade Kato², Hiromi Yuki² and Masayoshi Kurachi ${ }^{2}$

1. Tokyo Metropolitan Rehabilitation Hospital, Sumida-ku, Japan

2. Toyama Medical \& Pharmaceutical University, Toyama-shi, Japan

Objectives: This study is aimed at analyzing factor constructs of neuropsychological function of normal people.

Methods: Fifty-five healthy subjects (mean age $=24.9$ years, ranging from 16 to 40 years) were screened by both the Minnesota Multiphase Personality Inventory (MMPI) and the questionnaire for psychiatric settings to rule out psychiatric, drug, and alcohol problems (SCID), which was then followed by administration of the Digit Span, Picture Completion, Block Design, Vocabulary, Digit Symbol (Wechsler Adult Intelligent ScaleRevised), Logical Memory, Visual Reproduction (Wechsler Memory ScaleRevised), Wisconsin Card Sorting Test (WCST), Trail Making Test (TMT) A \& B, prospective memory task, Letter Number Sequencing (LNS), Word fluency test, and Japanese verbal learning test (Matsui et al., 2001). A factor analysis on the 15 variables of neuropsychological tests was performed using least squares solution and varimax rotation. The method of choosing components with an eigen value $>1$ was used as a guide. 
Results: Factor analysis indicated five factors: the first comprising TMTA, TMTB, and Prospective Memory named cognitive planning. The second comprising Digit Span, verbal learning test, LNS named short-term memory. The third comprising Vocabulary, Visual Reproduction, and WCST named conceptual image organisation. The fourth comprising Block Design and Picture Completion named visual spatial organisation and the fifth comprising Logical Memory, Word Fluency test and Digit Symbol named verbal memory organisation.

Conclusion: Further study is necessary to compare the factor constructs of neuropsychological function between normal people and patients with schizophrenia with reference to brain structure or brain function.

\title{
73237: Executive Functioning: The Development of Theoretical Bases
}

Maria Hennessy ${ }^{1,2}$ and Gina Geffen ${ }^{2}$

1. Centre for the Mind, University of Sydney, Sydney, NSW, Australia

2. Cognitive Psychophysiology Laboratory, School of Psychology, University of Queensland, Brisbane, QLD, Australia

7 he validity of domain specific versus process fractionation theories of executive functioning was examined, with the aim of developing a theoretical basis for executive functioning. Two frameworks were selected. The first was a four construct theory based on a domain specific functional adaptation of Goldman-Rakic's experimental primate research. The second used Luria's stage and process theory of complex cognitive behaviour. A group of normal participants $(n=73)$ was recruited from an undergraduate university setting. Eleven neuropsychological tests were chosen according to four domain specific executive constructs on an a priori basis. Principal components analysis produced a four factor structure that accounted for approximately sixty percent of the total variance. However, contrary to the hypothesis, process fractionation theory was a superior explanation for the latent structure within the data. The study provided empirical evidence for a process fractionation structure of executive functions, and experimentally defined four separable executive processes: checking, inhibiting, sharing and integrating. The process fractionation structure supported in this study needs to be validated and extended to include other potentially separable executive processes. This research provides evidence for the need to conduct theoretically derived and empirically validated research to advance an understanding of the structure of executive functioning.

\section{0 : Examination of the Clinical Utility of Cognitive Patterns in a Mixed Diagnostic Sample on the WAIS-III/WMS-III and Other Neuropsychological Tests}

\author{
Sharron E. Dawes ${ }^{1}$, Graeme J. Senior ${ }^{1,4}$, Rael T. Lange ${ }^{3}$ and Gordon Chelune ${ }^{2}$ \\ 1. Psychology, University of Southern Queensland, Toowoomba, QLD, Australia \\ 2. The Mellen Centre, Cleveland Clinic Foundation, Cleveland, OH, USA \\ 3. Riverview Hospital, Vancouver, BC, Canada \\ 4. Assessment Services in Psychology, Brisbane, QLD, Australia
}

fundamental assumption in neuropsychological assessment is that different disorders generate specific patterns of psychological test scores. Using cluster analysis, Lange (2000) challenged this assumption and presented compelling evidence that a small number of cognitive patterns were 
commonly found in a number of disorders on the WAIS-R/WMS-R. He derived three common cognitive profiles, which occurred with approximate equal frequency across seven disorders. Utilising the WAIS-III/WMS-III and other tests (including TMT, WCST, BNT, \& FAS), a nine-cluster solution of which five-clusters were retained, was derived using Hierarchical and K-Means cluster analysis. These clusters were determined to occur across all twelve diagnostic groups examined. If prototypical patterns exist related to diagnosis then stark asymmetries in the distribution of cluster membership should be evident across the diagnostic groups (e.g., profile for TBI should be distinct from the profile for CVA). The outlier metric, Mahalanobis Distance, was then used to allocate individual cases to one or more of these stable cognitive patterns in order to examine the clinical utility of this classification method.

\section{2: An Adaptive Approach to Psychological Test Battery Selection}

\section{Tammie Olm and Graeme J. Senior}

Psychology, University of Southern Queensland, Toowoomba, QLD, Australia

$\mathrm{O}_{a}^{\mathrm{n}}$ ne of the challenges confronting clinicians engaging in psychological assessment is how to combine information from a wide variety of assessment measures into a coherent system of interpretation. Assessors are often called upon to vary their psychological test batteries to better meet the limitations of their clients and address hypotheses regarding the nature of potential deficits. While the reliability and validity of individual tests should be well established, the psychometric properties of flexible batteries as a whole are not usually known. The difficulties inherent in the substitution of various measures into an individual battery, therefore, include the potential introduction of error due to a lack of knowledge regarding the interrelationships between the substituted measures. Detailed knowledge of the statistical relationships between individual tests would allow the clinician to control for the error associated with combining them into a battery. Such knowledge would also facilitate the combination of individual tests of the same underlying cognitive construct into composite scores with a known and controlled margin of error and so facilitate accurate and efficient analysis of test results. This paper presents a systematic method for determining how to substitute measures within a structural framework based upon assessment of clinically salient cognitive constructs. The model will be presented along with the algorithms for combining different psychological tests and computing the psychometric properties of their resultant composites. The study aims to present a "model" by which the clinician could combine a varying and clinically appropriate battery of individual tests using knowledge of their statistical interrelationships to control for psychometric error. This method is proposed as a way of more effectively and flexibly assessing the cognitive strengths and weaknesses of brain-damaged individuals. 


\title{
73284: Forensic Neuropsychology and the MMPI-2:
}

I. Discriminating Between Neurologic and Psychiatric Conditions

Graeme J. Senior ${ }^{1,2}$ and Lucille A. Douglas ${ }^{2}$

1. Psychology, University of Southern Queensland, Toowoomba, QLD, Australia

2. Assessment Services in Psychology, Brisbane, QLD, Australia

$\mathrm{T}$ The second edition of the Minnesota Multiphasic Personality Inventory (MMPI-2) is the most widely used measure of psychosocial maladjustment in the assessment of personal injury claimants. While initially developed for use in the assessment of psychiatric disorders, more and more, it is being used to examine those with neurological injuries and contribute to discriminating between neurological and psychiatric influences on psychosocial functioning. Utilising more than 3000 cases, this study examines the ability of the MMPI-2 to discriminate between personal injury claimants diagnosed with either neurological or psychiatric conditions. In particular, the role of the Basic and Content scales will be contrasted with those of measures developed for the specific assessment of neurological conditions.

\section{1: Forensic Neuropsychology and the MMPI-2: V. Development of a Structural Summary Approach to the Assessment of Personal Injury Claimants}

\author{
Hong Eng Goh and Graeme J. Senior \\ Psychology, University of Southern Queensland, Toowoomba, QLD, Australia
}

\begin{abstract}
$\mathrm{A}$ number of approaches have been proposed with regard to analysing and interpreting MMPI-2 protocols in order to assess psychosocial maladjustment. The current study presents a Structural Summary approach to the organisation and interpretation of the MMPI-2 developed specifically in the forensic setting and the assessment of personal injury claimants. The Structural Summary is based on a scale level principle components analysis with oblique rotation of more than 3000 MMPI-2 protocols. While ten components were derived, only eight form the basis of the structural summary. One component was represented by only a single scale while another was based on scale loadings (Mf, FRS2), the clinical significance of which remains undetermined. The utility of the approach will be demonstrated with illustrative case examples.
\end{abstract}

\section{7: WAIS-III Performance in Forensic Psychiatry Inpatients with Schizophrenia Spectrum Disorders}

Hendre Viljoen ${ }^{1}$, Grant Iverson ${ }^{2,1}$ and Johann Brink ${ }^{1,2}$

\footnotetext{
1. Forensic Psychiatric Hospital, Vancouver, BC, Canada

2. University of British Columbia, Vancouver, BC, Canada

3. Riverview Hospital, Vancouver, BC, Canada
}

Grom a clinical and commonsense perspective, some might hypothesise F that forensic psychiatric patients are likely to be more cognitively impaired than civil psychiatric patients. Forensic psychiatric patients typically have a history of violent behaviour, and they have relatively high rates of co-morbid substance abuse. Moreover, a history of traumatic brain injury also is common in this patient group. The purpose of this study was to compare the WAIS-III performance of a sample of forensic psychiatry inpatients with schizophrenia spectrum disorders to a non-forensic outpatient sample 
from the literature. It was hypothesised that the forensic psychiatry inpatients would have lower index scores than non-forensic outpatients given their relatively low education and frequent co-morbid substance abuse problems. The primary sample consisted of 30 patients from a 211 bed forensic psychiatric hospital in British Columbia, Canada. Their average age was 32.3 years $(S D=10.1)$. Their average education was 10.3 years $(S D=2.1)$. All patients had a diagnosis of one of the schizophrenia spectrum disorders. The patients demonstrated medium to large deviations from the healthy normative mean on the Verbal Comprehension Index $(M=91.5, d=.54$, medium effect size), Working Memory Index $(M=89.2 ; d=.69$, mediumlarge effect size), and the Processing Speed Index ( $M=82.3, d=1.22$, large effect size). The sample performed normally on the Perceptual Organisation Index $(M=98.3, S D=16.0)$. Contrary to expectations, the forensic sample did not differ on any of the WAIS-III index scores from a large sample of outpatients from Maryland with schizophrenia spectrum disorders (all $p \mathrm{~s}>$ $.05 ; N=120$; Dickson, Iannone, \& Gold, 2002), despite significantly less education in this forensic sample. This study suggests relative consistency of neurocognitive deficits in heterogeneous patients with schizophrenia spectrum disorders.

\section{3: The Relationship of the Problem Checklist and Competency Rating Scale to the MMPI-2 within a Forensic Setting}

Sharron E. Dawes ${ }^{1}$, Graeme J. Senior ${ }^{1,2}$ and Lucille A. Douglas ${ }^{2}$

1. Psychology, University of Southern Queensland, Toowoomba, QLD, Australia

2. Assessment Services in Psychology, Brisbane, QLD, Australia

The Problem Checklist (PCL; Cavallo, Kay, \& Ezrachi, 1992) and Competency Rating (CRS; Prigatano et al., 1986) scales were developed for the assessment of traumatic brain injury (TBI). While used primarily in the rehabilitation setting, recent research has suggested that they may be valuable in the assessment of the types of problems experienced by personal injury claimants with or without TBI, as well as the degree to which these difficulties impact upon their daily functioning. In contrast, the Minnesota Multiphasic Personality Inventory-2 (MMPI-2), while the most frequently used psychosocial inventory in the medicolegal setting, is limited by its mode of evaluation. Respondents on that test indicate only whether a statement is "True" or "False". Consequently, assumptions regarding type and severity of symptoms are based solely on the number of endorsements and not on any direct measure of perceived severity or frequency. The current study examines the relationship between the PCL and CRS which require respondents to indicate the level of perceived difficulty or severity they experience, and their responses on the MMPI-2. Additionally, the degree to which these measures characterise the type and extent of psychosocial difficulties of both TBI and non-TBI litigants will be examined. 


\section{3: Color Trails and Five-Digit Tests: Education Effects in Modern Greek Speakers}

Wendy Walker ${ }^{1}$ and Michael L. Drexler ${ }^{1,2}$

1. The Wright Institute, Berkeley, CA, USA

2. NHCU, San Francisco VA Medical Center, San Francisco, CA, USA

The Five-Digit Test (FDT) is a new instrument designed to assess areas of speeded or controlled information processing (Sedo, 1999). One subtest is designed to measure an interference effect, comparable to that observed in the Stroop Color-Word Test (Stroop,1935). However, the FiveDigit Test was designed to reduce reliance on vision and knowledge of numbers and letters. Thus, its utility for cross-cultural assessment has been noted, and it has been administered in English, Spanish, and Chinese (Lang, 2002). The contribution of important demographic variables to neuropsychological test performance is well known (Lezak, 1995), with education being important. The purpose of this study was to examine the influence of education across scores on the Five-Digit Test and to compare the results to an established cross-cultrual measure like the Color Trails Test in a Modern Greek-speaking sample. Subjects included 49 individuals recruited utilising a snowball sampling technique in Greece. The average educational level was 17 years $(S D=4)$. The complex relationship between education and performance across the subtests of the Color Trails Test and the Five-Digit Test was explored and a preliminary examination of normative implications was conducted. The development of this brief, easy to administer test may be relevant to those assessing patients in linguistically and culturally diverse areas such as Australia. The test was well received by all subjects. Suggestions for future research are offered, and the clinical utility of the test for cross-cultural assessment is discussed.

\section{8: Towards the Development of Alternate Prose Recall Passages}

\section{Karen Sullivan}

QUT, Carsdeline Campus, Carseldine, QLD, Australia

$\mathrm{R}$ epeat assessments of memory function can contribute to diagnosis and treatment evaluation, yet memory tests may be susceptible to practice effects. To reduce such effects it has been suggested that alternate forms of memory tests should be used where possible. In this study, alternate forms of the logical memory stories were devised. Passages were matched to the original Wechsler Memory Scale-Revised stories in terms of a number of passage attributes such as story length and readability. Three clinical neuropsychologists were asked to independently score taped passage responses to determine inter-rater reliability statistics. Estimates of passage difficulty were based on the responses of thirty-two undergraduate students asked to recall passages administered in a counterbalanced order. Preliminary investigations of the psychometric properties of prose recall stories devised in this study suggest these passages have good inter-rater reliability and are equivalent in terms of difficulty. Importantly, although the development of alternate prose recall passages is not new, the passages devised in this study have been shown to match each other across a broader range of variables than has been attempted previously and as such these passages may prove useful in the repeat assessment of auditory-verbal memory. 


\section{7: A Component Analysis of the Zoo Map Test}

Ian Burgess ${ }^{1}$, Dodd Amy ${ }^{1}$ and lain Gilchrist ${ }^{2}$

1. Psychology, Avon \& Wiltshire Mental Health Partnership Trust, Bristol, United Kingdom 2. Experimental Psychology, University of Bristol, Bristol, United Kingdom

The purpose of this research was to investigate the utility of the separate component scores of the Zoo Map 1 and 2 subtest of the Behavioural Assessment of Dysexecutive Syndrome battery relative to the usual Profile Score. As part of a neuropsycholgical assessment the Zoo Map test was administered to a group of clinical patients diagnosed with mental health problems. Their scores were compared with those of a control group. In addition to calculating the usual Profile Score we also analysed scores on Planning Time, Total Time, Action Time (Total Time - Planning Time), Sequence Score and Number of Errors across both Zoo Maps 1 and 2. Statistical analysis revealed that the clinical group had significantly lower Profile Scores than the control group. On Zoo Map 1 the clinical group had significantly shorter Planning Times (indicating online planning) but longer Action Times (indicating poorer sequencing ability) than the control group. On Zoo Map 2 Action Time was significantly longer for the clinical group. We conclude that the component scores provide more information as to how the different groups approach this test relative to the usual Profile Score. Implications for use of this test in clinical situations is discussed.

\section{2: The Twelve Elements Test of Executive Function}

Claire S. Sira, Roger Graves and Catherine Mateer

Psychology, University of Victoria, Victoria, BC, Canada

The Six Elements Test (SET; Shallice and Burgess, 1991) is a measure of planning and organisation. A potential difficulty with the SET is that there is a limited range (0-6) in possible scores. Because of practice effects, a test with limited range may lead to ceiling effects. A possible solution to this dilemma is increasing the number of tasks to increase the potential range of the task. This study reports initial results for our new modification of the SET, the Twelve Elements Test, which has a possible range of 0-12. This test was administered to 45 female and 12 male undergraduates (aged 17-26 years) with no history of brain injury or neurological condition. Results for this high functioning sample showed a good distribution of performance: Tasks Attempted $M=9.00, S D=2.74$, range 3 to 12; Number of subtask Switches $M=9.02, S D 3.47$, range 2 to 15 . This sample rarely broke the rule of the task $(M=0.26, \mathrm{SD}=0.67)$ and made few requests for the amount of time remaining $(M=1.51, S D=1.24)$. The relatively low mean and large range for tasks attempted suggest that our new test may better allow for reliable change to be calculated despite expected practice effects. Further work will determine the potential utility of this new test for evaluating executive dysfunction in a brain injured sample. 


\title{
73431: One-hand Versus Two-hand Tactile Bisection in Normal Subjects
}

Sue J. Kang ${ }^{1}$, Byung H. Lee', Min J. Baek² and Duk L. Na'

1. Neurology, Samsung Medical Centre, Sungkyunkwan University School of Medicine, Seoul, South Korea

2. Neurology, Seoul National University Bundang Hospital, Sungnam, South Korea

Background and Objectives: When asked to bisect a line presented through the visual modality, normal subjects tend to bisect slightly to the left of the true midpoint, a phenomenon termed "pseudoneglect". Tactile bisection also shows the pseudoneglect which, however, is less consistent among studies than in visual bisection. This inconsistency may be related to such factors as manual motor exploration, memory of the starting and ending position of the stimulus, or "overshoot phenomenon". This study investigated whether the pseudoneglect occurs even in a new tactile bisection task that minimises these factors.

Methods: Normal subjects were asked to bisect the distance (three different lengths) that spans between the right and left index finger by moving either of the fingers toward the midpoint. When moving the finger, in one condition the finger was to keep touching the surface of the stimuli (touch condition), and in the other the finger was moved in the air without touching the stimuli (jump condition). Thus, the experiment consisted of 12 conditions (three lengths $\mathrm{x}$ two hands $\mathrm{x}$ touch versus jump). Each condition had two trials and the total 24 trials were presented in a random order.

Results: Subjects were accurate in the jump condition but undershot in the touch condition. Conclusion: In the jump condition of the new version of tactile bisection, pseudoneglect did not occur, which suggests that pseudoneglect is restricted to the visual modality. Results of the touch conditions suggest that the surface resistance of the stimulus can be another variable that affects the tactile bisection performances of normal subjects.

\section{5: Modified Scoring System: Measurement of the Global and Local Elements of the RCFT in Children with and without ADHD}

\author{
Ramona A. Raggl', Sabine A. Wingenfeld ${ }^{1,2}$ and Sheila G. Crewther ${ }^{1}$ \\ 1. School of Psychological Science, La Trobe University, La Trobe University, VIC, Australia \\ 2. CAMHS, Austin Health, Heidelberg, VIC, Australia
}

Similar to most complex shapes, the Rey Complex Figure Test (RCFT) consists of global (the whole object in its entirety) and local features (the shapes embedded within the whole figure). As the RCFT has a high level of complexity, numerous scoring systems have been developed. No system independently measures the global components separate from the local elements rather they produce a single accuracy score assessing a combination of global and local features, and/or an organisational strategy score. A modified scoring protocol, termed Rey Complex Figure Global Organisation and Local Accuracy (RCF-GOLA), was designed to measure children's reproduction of the key local and global features. RCF-GOLA was based upon the theoretical premise of Taylor (Spreen \& Strauss, 1998) and the developmental perspective of Waber and HolmesBernstein (1996), thus measuring internal and external accuracy and developmental organisation. Reproductions of the RCFT (copy condition) by 210 children, including 20 children with ADHD, were scored according to the RCF-GOLA and compared to two other scoring systems. 
Results revealed high inter-scorer reliability, good concurrent validity with the other scoring systems, sensitivity to gender and developmental trends, and discrimination between children with and without ADHD. In conclusion, the RCF-GOLA scoring protocol accurately assessed higherorder developmental perceptual and executive functioning impairments. The RCF-GOLA can contribute to our understanding of paediatric global and local processing performance, and may be useful in the assessment of childhood executive and perceptual disorders.

\section{8: Faking It! The Use of a Battery of Tests to Detect Malingered Amnesia}

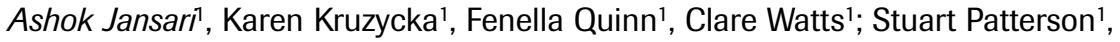
Anna Cantagallo ${ }^{2}$, Alessandro Maietti², Manuela Maini², Angela Gosling ${ }^{1}$

1. School of Psychology, University of East London, London, United Kingdom

2. Department of Clinical Neuropsychology, Unita di Medicina Riabilitativa, Ferrara, Italy

$\mathrm{T}$ he malingering of amnesia is reported to be increasing in society; despite this, methods to detect it successfully still do not exist. Studies attempting to detect malingered amnesia have found that when using single tests, although detection rates of participants instructed to simulate can be significant, misclassification of individuals still occurs. Additionally, knowledge provided to simulators has been shown to have equivocal effects on performance. A series of experiments evaluated the combined ability of three neuropsychological tests to accurately identify whether or not an individual is feigning memory impairment; coaching given to simulators was also manipulated. The performance of three groups (naive simulators, coached simulators and controls) was measured across the tests used by Hanley et al. (1999): word fragment completion, coin-in-the-hand and distraction/no-distraction tests. Discriminant function analysis on accuracy scores resulted in $85.4 \%$ of individuals being classified accurately. A translation of the paradigm into Italian resulted in $86.7 \%$ accurate classification. Finally, an extension of the paradigm using computerised presentation such that reaction times as well as accuracy could be recorded resulted in an $81.9 \%$ success rate. The findings suggest that the pattern across tests is useful in discriminating between malingerers and genuine amnesics. The results also suggest that even when individuals are given information regarding the pattern of impairment of amnesia they cannot use this effectively to avoid detection. The practical implications of the findings as well as further directions for research are discussed.

\section{4: Utility of Hospital Anxiety and Depression Scale on Cross-Cultural Traumatic Brain Injured Patients}

Samir Al-Adawi', Atsu A. Dorvlo², Mel B. Glenn³, David T. Burke ${ }^{3}$ and Elizabeth Selleck ${ }^{4}$

1. Behavioural Sciences, Sultan Qaboos University, Muscat, Oman

2. Department of Mathematics and Statistics, Sultan Qaboos University, Muscat, Oman

3. Department of Physical Medicine and Rehabilitation, Harvard Medical School, Boston, MA

4. Department of Research and Training, Harvard Medical School, Boston, MA

The detection of mood disturbance is of great clinical importance in the rehabilitation of patients who have endured traumatic brain injury. Imported assessment measures from western cultures have not been validated in other cultures. This study assesses the Hospital Anxiety and 
Depression Scale (HADS). Forty-seven male and twenty-one female patients were screened using HADS and interviewed with the semi-structured, Composite International Diagnostic Interview (CIDI), in order to investigate the relationship between false positives and negatives at different cut-off points of the HADS. A receiver operating characteristic curve was used to discriminate the power of the HADS for every possible threshold score. Nine percent of the patients were identified by HADS as probable anxiety cases and nineteen percent as probable depression cases, compared to $50 \%$ and $57.4 \%$ respectively by the structured interview based on the gold standard. The sensitivity and specificity for the anxiety subscale of HADS were $17.6 \%$ and $97.1 \%$ respectively, while the sensitivity and specificity for the depression sub-scale were $33 \%$ and $100 \%$. In using the ROC analysis, the cut-off score of 5 gave the best compromise between sensitivity, $61.8 \%$, and specificity, $61.8 \%$, for anxiety, and the cut-off score of 4 gave the best compromise between sensitivity, 53.8\%, and specificity, $75.9 \%$, for depression. The results are discussed with respect to the concept of depression from cross cultural settings and impaired goal-directed behaviour in patients with traumatic brain injuries.

\title{
73216: Structure of the Visual \& Object Space Battery (VOSP)
}

\section{Clive Skilbeck}

Psychology, University of Tasmania, Hobart, TAS, Australia

\begin{abstract}
Warrington \& James' Visual Object \& Spatial Perception battery (VOSP; 1990) has been available to clinicians for 12 years, although studies on its structure are rare. The present research provides the first factor analytic study of the VOSP carried out on a patient sample (subarachnoid haemorrhage; $\mathrm{SAH}$ ). The data obtained from 76 participants suggest a 3factor structure (Object Recognition, Spatial Coordinates, Relative Position). The study also included correlation of these factors with other tasks (WAIS-R subtests, complex figure). Whilst the Object Recognition and Spatial Coordinates factors showed significant correlations with a number of these tasks, Relative Position only correlated significantly with Mental Arithmetic. This finding possibly reflects a shared visual imagery aspect of the two measures. Only non-significant effects upon the three factors were noted from gender and age.
\end{abstract}

\section{1: The Shipley Institute of Living Scale: Australian Normative Data and Clinical Utility}

Sharron E. Dawes ${ }^{1}$, Graeme J. Senior ${ }^{1,2}$ and Lucille A. Douglas ${ }^{2}$

1. Psychology, University of Southern Queensland, Toowoomba, QLD, Australia

2. Assessment Services in Psychology, Brisbane, QLD, Australia

The Shipley Institute of Living Scale (SILS) was originally developed by Walter Shipley in 1940 as a test designed to provide a brief assessment of an individual's level of intellectual functioning. The SILS is a self-administered test and consists of two main components: Vocabulary and Abstraction. The Vocabulary component consists of 40 items and the examinee is required to choose the best synonym from among four words. The Abstraction component consists of 20 items that requires the examinee to complete a logical sequence of numbers, letters, or words. Traditionally, these measures are used to compute six summary scores: Vocabulary score; 
Abstraction score; Total Score; Conceptual Quotient; Abstraction Quotient; and an estimated WAIS-R Full Scale IQ. The current study will present data from more than 400 participants collected as part of normative studies at the University of Southern Queensland, Toowoomba, Australia. This normative data updates not only the norms to include WAIS-III FSIQ, VIQ, GAI, and VCI but substantially reduces the complexity of the scoring analyses to reflect more contemporary approaches to discrepancy analysis. The utility of this approach will be demonstrated in a sample of clinical cases derived primarily from medicolegal practice.

\title{
73437: Revised Norms for the Raven's Coloured Matrices for Australian Primary School-aged Children
}

Sue M. Cotton ${ }^{1,2}$, Sheila G. Crewther ${ }^{2}$, Patricia Kiely², David P. Crewther ${ }^{3}$, Vito Milana ${ }^{2}$, Brenda Thomson ${ }^{2}$ and Robin Laycock ${ }^{2}$

1. ORYGEN Research Centre, Department of Psychiatry, University of Melbourne, Parkville, VIC, Australia

2. School of Psychological Science, La Trobe University, Bundoora, VIC, Australia

3. Brain Sciences Institute, Swinburne University of Technology, Hawthorn, VIC, Australia

The Raven's Coloured Progressive Matrices (CPM) has commonly been employed as a measure of nonverbal intelligence or the nonverbal component of Spearman's g factor, particularly in the United Kingdom and in Australia. The last Australian norms for the CPM were published over two decades ago and can be considered outdated. The aim of this study was to generate new CPM norms for a sample of 534 Australian children (males $n$ $=273$, females $n=258$, ranging in age from 6 to 12 years) and to contrast these findings to the previous standardisation. Significant changes in CPM performance were noted with age, however, no gender differences were evident. Hence, percentile ranks were calculated for the six age levels. There were minimal, if any changes, between the previous Australian norms and the current study, indicating that Australian children's performances on the CPM have remained relatively stable over time.

\section{4: Impact of Cultural Content of Verbal Abilities Testing}

Suzanne Barker-Collo

Dept of Psychology, University of Auckland, Auckland, New Zealand

\begin{abstract}
A ssessment of verbal abilities (e.g., naming and verbal memory) is a core component of neuropsychological assessment. As with other neuropsychological tests, naming and memory tasks may be impacted by cultural relevance of test content. This poster presents the findings of two studies examining the impact of American content on performance on New Zealanders. Study 1 examined whether 58 New Zealand students perform differently on the Boston Naming Test (BNT) when compared to available North American norms. Mean performance of the sample was significantly worse than norms $(F(171)=55.296, p<.01$, placing the average New Zealand participant 1.2 SDs below the mean. New Zealanders made $60 \%$ more error on the items pretzel and beaver, and $20 \%$ more errors on globe, funnel, and tripod than North Americans. Study 2 examined the extent to which the American content of the California Verbal Learning Test (CVLT) impacts verbal memory performance of $90 \mathrm{New}$ Zealand adults aged 17 to 81. Participants completed the CVLT and a version of this test modified to reflect New Zealand content (NZ-VLT). Within-subject comparisons
\end{abstract}


revealed that participants performed significantly better on the NZ-VLT with CVLT performance placing the average New Zealander between 1.0 and 1.5 SDs below the mean for long delay free recall and just below 1.0 SD below the mean for long delay cued recall. Conclusion: It was concluded that in administering these tests of verbal abilities to New Zealanders attention should be given to the potential for cultural biases.

\title{
72912: Accuracy of the Wechsler Test of Adult Reading (WTAR) as an Estimate of (Premorbid) IQ in a Healthy Australian Sample
}

\author{
J.L. Mathias ${ }^{1}$, M. Barrett-Woodbridge ${ }^{1}$ and S. Bowden ${ }^{2}$ \\ 1. Department of Psychology, University of Adelaide, Adelaide, SA, Australia \\ 2. Department of Psychology, University of Melbourne, Melbourne, VIC, Australia
}

\begin{abstract}
$\mathrm{M}$ easures of premorbid IQ are commonly used by clinicians to evaluate changes to the cognitive performance of patients with a wide variety of neurological disorders. The recently released WTAR has been co-normed with the third editions of the Wechsler Adult Intelligence and Memory Scales (WAIS-III, WMS-III), and has been shown to provide reliable and valid estimates of premorbid IQ in American and English samples. However, the suitability of the WTAR for use with an Australian population has not been established, nor is there any clear basis for determining whether to use American or English norms to convert WTAR raw scores to estimates of premorbid intellectual ability. This study examined the accuracy with which both the WTAR and the National Adult Reading Test (NART) estimated WAIS-III IQ performance in a sample of 50 healthy Australian adults. WTAR reading, demographic, and combined reading plus demographic estimates of WAIS-III VIQ were calculated using both US and UK norms, as was a NART reading estimate of WAIS-R VIQ. Preliminary analyses revealed that although the WTAR reading and the combined reading plus demographics estimated VIQ scores were correlated with WAIS III VIQ scores (US and UK norms), there were significant differences between estimated and actual WAIS-III VIQ scores. Demographic estimates of VIQ, based on WTAR normative data, were not significantly correlated with WAIS-III VIQ scores. Finally, NART estimates of WAIS-R VIQ also correlated with WAIS-III performance but yielded smaller differences between estimated and actual VIQ scores. All estimates of IQ underestimated WAIS III performance to varying degrees. These findings are discussed in terms of their implications for selecting a measure (WTAR versus NART) and method (WTAR reading, demographic, or reading plus demographic information) for estimating premorbid IQ in clinical settings and for making choices about which norms to use (WTAR US or UK norms).
\end{abstract}




\title{
POSTER SESSION 3: CHILDHOOD DISORDERS, OCD, ADHD AND LEARNING DISABILITIES, DEVELOPMENTAL STUDIES, PSYCHIATRIC CONDITIONS
}

\section{1: Motor Correlates of ADHD: Contribution of Reading Disability and Oppositional Defiant Disorder}

\author{
Libbe Kooistra ${ }^{1,2}$, Susan G. Crawford ${ }^{2}$, Deborah Dewey ${ }^{1,2}$, Marja Cantell1,2 \\ and Bonnie J. Kaplan ${ }^{1,2}$ \\ 1. Paediatrics, University of Calgary, Calgary, AB, Canada \\ 2. Behavioural Research Unit, Alberta Children's Hospital Research Centre, Calgary, AB, Canada
}

\begin{abstract}
$\mathrm{T}$ he study investigated (1) whether motor impairment in Attention Deficit Hyperactivity Disorder (ADHD) increases as a function of co-occurring disorders, (2) whether the co-occurring diagnoses of reading disability (RD) and oppositional defiant disorder (ODD) account for the motor deficits seen in ADHD. A total of 291 children (218 boys, 73 girls) participated. Five groups of children: ADHD-only $(n=29)$, RD-only $(n=63)$, ADHD and RD $(n=47)$, ADHD and ODD $(n=19), \mathrm{ADHD}$ and RD and ODD $(n=21)$ were compared with normally developing comparison children $(n=112)$. Motor skills were assessed with the Bruininks Oseretsky Test of Motor Performance, the Beery Visual Motor Test of Integration, and the Southern California Motor Accuracy Test. Key findings were the following: (1) the motor skills of the ADHD-only group did not differ from the comparison group overall; (2) motor impairment in ADHD increased as a function of co-occurring disorders; and (3) RD status rather than ADHD status predicted motor impairment.
\end{abstract}

\section{6: Using a Continuous Performance Test to Measure Sustained Attention in Preschoolers at Risk for ADHD}

Rachel L. Mountjoy ${ }^{1}$, Sabine A. Wingenfeld ${ }^{1,2}$, Anna-Karin Ohlen ${ }^{1}$ and Voon Seem (Jane) Khoo ${ }^{1}$

1. School of Psychological Science, La Trobe University, La Trobe University, VIC, Australia

2. Child and Adolescent Mental Health Service, Austin Health, Heidelberg, VIC, Australia

Dreschool-age children displaying high levels of inattentive and hyperacP tive behaviour may be at risk for the development of Attention Deficit Hyperactivity Disorder (ADHD). The primary impairment in school age children with ADHD is regarded to be a deficit in the ability to sustain attention, which has predominantly been assessed by Continuous Performance Tests (CPTs). Sustained attention in preschool children at risk for ADHD has comparatively been under-researched, despite the potential for CPTs to assist with the early detection of symptoms of ADHD. The present study aimed to examine the utility of CPTs in preschoolers and to investigate the relationship between parent-reported attention and activity level with sustained attention performance. Sixty-six preschool children were administered a CPT and parents completed ratings of their child of hyperactive and inattentive behaviours. Fifteen percent of the sample were classified as at risk for ADHD. Results indicated that overall performance on the CPT could not predict levels of parent-reported hyperactivity and inattention in preschool children. Problems with sustaining attention were found in children at risk, with higher levels of inattention and hyperactivity for CPT omission errors only. CPT performance did significantly predict observed 
inattentive and hyperactive behaviour during the task. The CPT was found to be an appropriate measure of sustained attention for use in preschool children as young as three years of age. Developmental effects were evident with performance on the task improving with age. Omission errors were found to be the best discriminator of performance. Length of the inter-stimulus interval affected overall performance on the CPT.

\section{2: Development of Response Inhibition and Interference Control and Relationship to Inattention and Hyperactivity in Preschoolers}

Anna-Karin Ohlen', Sabine A. Wingenfeld ${ }^{1,2}$, Rachel L. Mountjoy' and Voon Seem (Jane) Khoo ${ }^{1}$

1. School of Psychological Science, La Trobe University, Bundoora, VIC, Australia

2. Child and Adolescent Mental Health Service, Austin Health, Heidelberg, VIC, Australia

The association between inhibition and ADHD is well established for school age children, however, much less is known about this relationship in preschoolers. The current study examined the development of two types of inhibition (response inhibition and interference control), the relationship with each other, and with inattention and hyperactivity, in a nonclinical sample of preschool children. Eighty-eight preschool children (3:1 to 5:7 years) completed a response inhibition task (Puppet Says) and an interference control task (NEPSY Statue). Parent ratings on the PIC-2 Early Childhood Questionnaire were used to assess inattention and hyperactivity. Results showed a significant modest association between the two inhibition tasks but this correlation disappeared when controlling for age and IQ. Reasonable levels of inhibition were found to have developed by the age of three, with older preschoolers demonstrating higher levels of inhibition. No significant gender differences were found. The relationship between the two types of inhibition and inattention/hyperactivity was not significant when controlling for age and IQ. Results are discussed with respect to theories of inhibition deficits.

\section{3: Duchenne Muscular Dystrophy: Evidence for Subgroups Based on Profiles on the Wechsler Intelligence Scales}

Sue M. Cotton ${ }^{1,2}$; Nicholas Voudouris ${ }^{2}$; Kenneth Greenwood ${ }^{2}$

1. ORYGEN Research Centre, Department of Psychiatry, University of Melbourne, Parkville, VIC, Australia

2. School of Psychological Science, La Trobe University, Bundoora, VIC, Australia

nomalies in cognitive functioning have commonly been observed in Achildren with Duchenne Muscular Dystrophy (DMD), and include impairments in attention, memory, language, and visuospatial and auditory processing. Not all children with DMD experience these problems, lending support for the argument that subgroups exist within the DMD population. The aim of this study was to employ meta-analytical data to establish whether subgroups within the DMD population can be identified using scores from the Wechsler Intelligence Scales (WIS). The sample was derived from 32 published studies and comprised data on 1146 children with DMD between the ages of 2 and 27 years. K-means cluster analysis defined three groups of children based on the WIS subscales. These groups varied in overall ability (ranging from very low to average functioning) and the extent to which verbal skills were compromised. Further analyses revealed that these groups also differed in age and severity of disease. These 
findings are discussed with reference to brain dystrophin and the neurogenetics of DMD.

\section{4: Cognitive Outcome in Children of Mothers with Phenylketonuria}

Signy Wegener', Pamela Joy ${ }^{1}$, Arthur Shores ${ }^{2}$ and Bridget Wilcken ${ }^{3}$

1. Developmental Cognitive Neuropsychology Research Unit, The Children's Hospital at Westmead, Sydney, NSW, Australia

2. Department of Psychology, Macquarie University, Sydney, NSW, Australia

3. Department of Newborn Screening and Biochemical Genetics, The Children's Hospital at Westmead, Sydney, NSW, Australia

We investigated a number of unresolved issues regarding the nature of the cognitive impairment caused by exposure to phenylalanine, a neurotoxin, in the prenatal period. To do so we studied 9 mothers with phenylketonuria (PKU) and their 13 children aged 4 to 17 years. The sample consisted of 9 mothers, and their 13 children aged 4 to 17 years. Both mothers and children were administered selected subtests of the WoodcockJohnson, 3rd Edition (WJ-III). Blood phenylalanine levels during pregnancy were obtained retrospectively from medical records. As expected, children exposed to average phenylalanine levels of greater than $360 \mathrm{mmol} / \mathrm{litre}$ in utero had a significantly poorer intellectual outcome than those children exposed to levels below $360 \mathrm{mmol} /$ litre. Contrary to expectations, maternal IQ but not exposure to Phe during pregnancy significantly predicted offspring cognitive outcome and alone accounted for approximately $68 \%$ of the variance in observed scores. Children's scores on the WJ-III indices were not significantly different, and therefore failed to provide evidence for a specific cognitive profile in MPKU. Implications of these findings for the treatment and management of PKU for women planning pregnancy are discussed, along with the strengths and limitations of the current results and directions for future research.

\section{2: Neuropsychological and Neurochemical Profiles in Childhood Adrenoleukodystrophy}

Elsa G. Shapiro', Gulin $\mathrm{Oz}^{2}$, Kendra Bjoraker ${ }^{1}$, Lawrence Charnas ${ }^{1}$ and Rolf Gruetter ${ }^{2}$

1. Pediatrics, University of Minnesota, Minneapolis, MN, USA

2. Radiology, University of Minnesota, Minneapolis, MN, USA

Cerebral ALD (adrenoleukodystrophy) is an X-linked white matter (WM) disease with usual onset between 7 and 10 years of age with rapid demyelination of posterior pathways $(85 \%)$ and a few with anterior $(15 \%)$, and death within several years. About half with the biochemical abnormality develop cerebral disease. No method exists except close monitoring with MRI and neuropsychological (NP) tests to identify the onset of cerebral disease. Hematopoietic cell transplant (HCT) is beneficial if done early enough, but has lacked benefit if the P IQ $<80$, necessitating early detection of cerebral change. We present preliminary results of a study of the contribution of high field proton Magnetic Resonance Spectroscopy (MRS) in heralding disease onset, following treatment efficacy, elucidating pathophysiology, and correlating with NP function. 15 boys, 5 to 11 years of age, with ALD, 10 with no cerebral disease were seen every 6 months for NP testing and MRS on a 4 Tesla magnet (watching videotapes instead of sedation during $40 \mathrm{~min}$. of acquisition). 5 boys were seen prior to HCT and yearly thereafter. Single voxel $(8-16 \mathrm{ml})$ spectra from occipital and frontal 
WM were obtained. 14 adults and 3 children were normal controls. Results indicated stable NP patterns in boys with no WM lesions. However, even small areas of demyelination in posterior regions result in decreased visual spatial ability relative to other functions. On MRS, 11 metabolites were measured reliably. Several metabolites were markers of lesions, and others normalised as a result of successful HCT (as contrasted to MRI which showed no change and NP tests which showed mild improvement). Comparing NP testing with lesion patterns before and after HCT, correlation was found between frontal lesions and executive, speed, and memory scores, while improving neurochemical profiles in occipital WM were correlated with visual perceptual scores. In sum, MRS is sensitive to changes in WM and correlates with NP status.

\title{
72888: Different Infant's Brain Activation for Mother and Stranger in Strange Situation: A Near-Infrared Spectroscopy Study
}

\author{
Takeo Kondou ${ }^{1,2}$, Tamotsu Toshima ${ }^{3}$ and Yukari Hashimoto ${ }^{3}$ \\ 1. Japan Foundation of Aging and Health, Chita, Japan \\ 2. Graduate School of Biomedical Sciences, Hiroshima University, Hiroshima, Japan \\ 3. Graduate School of Education, Hiroshima University, Higashi-hiroshima, Japan
}

\begin{abstract}
$\mathrm{N}^{-\infty}$ ear-infrared spectroscopy (NIRS) is a non-invasive technique that is able to measure relative changes of oxy $\left(\mathrm{C}^{\text {oxy }}\right)$, deoxy $\left(\mathrm{C}^{\text {deoxy }}\right)$ and total $\left(\mathrm{C}^{\text {total }}\right)$ hemoglobin in response to cortical brain activation. This technique is expected to be one of a number of useful methods to obtain infants' brain activation data which up to now have been few and far between. To investigate whether infants have an ability to distinguish between their mother and a stranger, we measured infants' brain activity in a strange situation by multichannel NIRS. This ability is also important for the mother-infant-bond. Participants were six infants (aged 3-9 months) and their mothers. A female assistant played the role of the stranger. In the relaxed state (no interaction with others) which we used as a baseline, we measured infants' relative hemoglobin changes in the occipital lobe induced by interaction between the infant and their mother or a stranger (i.e., A person talks to and amuses an infant face-to-face). When infants were interacting with others, $\mathrm{C}^{\text {oxy }}$ and $\mathrm{C}^{\text {total }}$ in their left hemisphere were significantly larger than in their right hemisphere. Interestingly, however, different hemodynamics for the mother and stranger were found in two channels in the right hemisphere. The amounts of relative hemoglobin changes for the stranger were larger than those for their mother. These differences were significant in $\mathrm{C}^{\text {oxy }}, \mathrm{C}^{\text {deoxy }}$ and $\mathrm{C}^{\text {total }}$ in the channel in parieto-lateral region and in $\mathrm{C}^{\mathrm{oxy}}$ in the channel in occipito-lateral region. These results suggest that (1) the situation of interacting with others increased left hemispheric cortical activation, which might reflect left-hemispheric superiority for language processing, (2) according to whether infants interact with their mother or a stranger, the right hemispheric cortical activations were different, which might reflect right-hemispheric superiority for specific object (face) recognition processing. It is considered that NIRS could measure brain activities involved in infants' ability to distinguish their mothers from strangers in a strange situation.
\end{abstract}




\title{
73369: Arithmetic Tasks Performance in 6- to 16-year-old Latin-American Children
}

Esmeralda Matute $^{1,2}$, Teresita Montiel2 ${ }^{2}$, Monica Rosselli ${ }^{3}$ and Alfredo Ardila ${ }^{4}$

1. Instituto de Neurociencias, Universidad de Guadalajara, Guadalajara, Mexico

2. Departamento de Estudios en Educacion, Universidad de Guadalajara, Guadalajara, Mexico

3. College of Liberal Arts, Division of Science, Florida Atlantic University, Davie, FL, USA

4. Communication Sciences and Disorders, College of Health and Urban Affairs, Florida International University, Miami, FL, USA

While it is known that culture has an effect upon several neurocognitive functions, knowledge is limited concerning the effect of age in the development of arithmetic domains among children from different cultures. Objective: To analyse the effect of age on several arithmetic tasks performance across 5- to 16- year- old Latin-American children, and to set up the correlations between arithmetic tasks and executive functions tasks.

Method: We analysed the performance of 248 Colombian and 540 Mexican children ( 349 boys and 439 girls) on nine arithmetic related tasks, three conceptual tasks and 7 executive functions tasks from the ENI (a Child Neuropsychological test for Hispanic children).

Results: An overall MANOVA (Hotelling's T) revealed a significant age effect over the arithmetic measures $(p<.001)$. Univariate ANOVAs and post-hoc Tukey analysis demonstrated that more accentuated changes among adjacent age occurred before age 10 years in each one of the arithmetic tasks. A Pearson correlation analysis showed a stronger correlation between arithmetic tasks and conceptual tasks that between the former and executive functions tasks.

Conclusions: An age effect was present over all the arithmetic tasks and was more accentuated before age 10 years. Even the simplest arithmetic tasks, such as counting, are related to concept formation and reasoning.

\section{4: Prediction of Problem Behaviour in Preschoolers from Language and Cognitive Measures}

\author{
Voon Seem (Jane) Khoo ${ }^{1}$, Sabine A. Wingenfeld ${ }^{1,2}$, Anna-Karin Ohlen ${ }^{1}$ \\ and Rachel L. Mountjoy' \\ 1. School of Psychological Science, La Trobe University, Bundoora, VIC, Australia \\ 2. Child and Adolescent Mental Health Service, Austin Health, Heidelberg, VIC, Australia
}

R esearch has suggested that behavioural difficulties seen in preschoolers may reflect undiagnosed underlying neurodevelopmental problems and delays. This study investigated the relationship between language and behaviour in a community sample of 85 preschool children aged three to five. Specific language and neurocognitive abilities - receptive and expressive language, verbal short term memory and phonological processing speed - were examined as predictors of parent-reported externalising and internalising behaviours observed in the children. Children completed a range of language and neurocognitive measures (NEPSY, WoodcockJohnson-III). Parent-report on behavioural adjustment was obtained with the PIC-2 Early Childhood Questionnaire. Positive linear relationships were found between cognitive and language abilities. Lower performance on language and cognitive measures were associated with higher levels of internalising and externalising behaviour. Verbal short-term memory was found 
to predict receptive and expressive language performance. Phonological processing speed predicted expressive language only. Lower performance on the language and cognitive measures was associated with more behavioural adjustment difficulty. Verbal short-term memory and phonological processing were comparable predictors of externalising behaviour. Internalising behaviour was more strongly related to phonological processing speed. The strength of language predictors for behaviour ratings remains unclear. Findings suggest that expressive language ability may influence both externalising and internalising behaviour more strongly than receptive language ability.

\title{
73500: Relative Stability of the Neuropsychological Profiles of NLD and BPPD from Middle Childhood to Early Adolescence
}

\author{
N.S. Koushik, B.P. Rourke and S.A. Ahmad \\ Psychology, University of Windsor, Windsor, ON, Canada
}

$\mathrm{T}$

The present study is a longitudinal comparison of relative neuropsychological assets and deficits for young and older children with Nonverbal Learning Disabilities (NLD) and Basic Phonological Processing Disabilities (BPPD). The sample for the investigation was comprised of clinic-referred children who had been diagnosed with either NLD or BPPD by an experienced clinical neuropsychologist. A total of 18 children, 9 in each of the NLD and BPPD groups were selected. The children were between 7 and 8 years of age at first testing, and 9 and 14 years of age at second testing, inclusive. The NLD and BPPD groups were compared across young and older ages on a number of achievement, verbal, visualspatial, motor/psychomotor, tactile-perceptual, and conceptformation/problem-solving measures. Results of the comparisons confirmed the pattern of relative neuropsychological assets and deficits as predicted by the NLD and BPPD models for both young and older children. An examination of age-related profiles of neuropsychological functioning suggested that the children with BPPD demonstrated age-appropriate development with regard to their neuropsychological deficits, but the children with NLD demonstrated a pattern of worsening with regard to some of their neurospychological deficits. Specifically, the performance of the children with NLD declined significantly in areas related to Arithmetic and Complex-Tactile abilities. Children in both LD subtypes demonstrated ageappropriate development of the majority of their respective neuropsychological assets. The failure to confirm all of the age-related predictions based upon the NLD and BPPD models, along with the neuropsychological implications of the findings, are discussed.

\section{5: Age-related Differences in Simple, Discrimination and Choice Reaction Time in Preschool Children}

Sergey Y. Kiselev

Clinical Psychology, Urals State University, Yekaterinburg, Russian Federation

The goal of this study was to investigate the rate of change of reaction time (RT) with respect to different RT tasks in preschool children using a computerised battery of nonverbal tasks. The study sample consisted of 54 4-years-olds, 52 5-years-olds, and 59 6-years-olds. There were notable differences between the 3 age groups in terms of median RTs. However, the 
age-related change of reaction time with respect to various RT tasks had different rates. The rate of change of simple RT, colour discrimination RT, and two-choice RT during the period between 4 and 5 years of age, and between 5 and 6 years of age was relatively equal. However, the rate of change of four-choice RT, spatial discrimination RT, and alteration two-choice RT during the period between 4 and 5 years of age was more than during the period between 5 and 6 years of age. The highest rate of change during the period between 4 and 5 years of age was observed for four-choice reaction time. The current study provides further support for the global development trend hypothesis with respect to processing speed. However it may be assumed that there are several reasons for developmental decreases in RT. Together with increasing of central processing speed, decreasing RT appears to be related to maturation of those areas of a brain which are actively involved in each type of RT task. These results may help explain the agerelated change in performance of various RT tasks in preschool children.

\title{
73158: Executive Functions and Relational Complexity in Normally Developing Children
}

\author{
Toni J. Jones, Graeme S. Halford and Ken McFarland \\ Psychology, University of Queensland, Brisbane, QLD, Australia
}

$\mathrm{T}$ he relationship between executive functioning and the ability to process relational information has long been recognised in psychology. Halford and his colleagues have developed a measure of relational (structural) complexity based on the number of independent units of information, which need to be related in a cognitive representation. The first level, known as unary relations requires representation of a single component in relation to attributional or categorical information, for example, big dog. Binary relations involve the representation of two entities and their relationship such as an elephant is larger than a dog. Ternary relations involve the representation of three elements and two binary relations, for example, Tom is taller than David, David is taller than Paul, therefore Tom is taller than Paul. Halford and his colleagues have identified developmental changes in performances on tasks which vary only in terms of the complexity of relational information to be processed, with most five-year-olds succeeding on tasks requiring ternary level processing. Other researchers have identified similar developmental changes in executive functioning with significant changes occurring in preschool years. The present paper reports on an investigation of the relationship between performances on executive function and relational complexity tasks in 108 normally developing children between the ages of 3:6 and 8:6. Results of regression analyses indicate performances on ternary-level tasks predict a significant amount of unique variance on executive function tasks over and above that associated with age and verbal intelligence. The implications of these findings in terms of current models of executive functioning and possibilities for future research will be discussed. 


\title{
72128: Nonverbal Learning Disabilities in Children with Hydrocephalus
}

Knut Dalen', Sidsel Bruaroy², Tore Wentzel-Larsen ${ }^{3}$ and Liv Laegreid ${ }^{2}$

1. Biological and medical psychology, University of Bergen, Bergen, Norway

2. Child Neurology and Habilitation, Haukeland University Hospital, Bergen, Norway

3. Centre for Clinical Research, Haukeland University Hospital, Bergen, Norway

The aim of the present study is to investigate nonverbal learning disabilities (NLD) in children with hydrocephalus (HC). The Neuropsychological Assessment of Children (NEPSY) was administered to 57 children with $\mathrm{HC} ; 39$ boys and 18 girls, and to 44 controls, 27 boys and 17 girls. The controls were matched according to age, gender, and geographic variables. Age group 4-8 years, born between 1989-1996. All children were living in western Norway. 40 children had congenital $\mathrm{HC}$ and 17 had acquired HC. In order to make the groups as homogenous as possible, children with spina bifida, brain tumor, mental retardation (IQ $<70$ ), or a foreign mother language were excluded. The NEPSY subtests were classified along each of the dimensions "assets" or "deficits", according to Rourke's model of the elements and the dynamics of the NLD syndrome. Based on Korkman's description of the NEPSY subtests, five subtests were classified as measuring functions described by Rourke as "assets", whereas 16 subtests were classified as measuring "deficits". Differences between sumscores for the subtests classified as "assets", versus "deficits" were compared (by a permutation test) between the groups (HC and controls). The results demonstrated a significantly higher difference between "assets" and "deficits" in the HC group as compared with the controls $(p<0.001)$, compatible with a higher frequency of NLD in the HC group. As such, the model of the elements and the dynamics of the NLD syndrome, as described by Rourke, may be useful when analyzing neuropsychological test profiles.

\section{6: Specificity and Characteristics of Learning Disabilities}

\author{
Natasha Eisenmajer ${ }^{1}$, Nola Ross ${ }^{2}$ and Chris Pratt ${ }^{1}$ \\ 1. School of Psychological Science, La Trobe University, Melbourne, VIC, Australia \\ 2. Learning Difficulties Clinic, Sunshine Hospital, Melbourne, VIC, Australia
}

$\mathrm{T}$

The specificity of impairments in specific reading disabilities (SRD) and specific language impairments (SLI) has recently been questioned, with many children recruited for studies of SRD and SLI demonstrating impairments in both reading and oral language development. This has implications for the results of previous SRD and SLI studies in which reading and oral language skills have not been assessed. Thus there is a need to compare the profiles of children with mixed oral language and reading impairments to groups of children with SRD and SLI. The reading, oral language, shortterm auditory memory, phonological processing, spelling, and maths abilities of 151 children (aged between 7 and 12 years) drawn from a Learning Disabilities Clinic were assessed. Five groups were identified, and although some children demonstrated a specific reading disability or a specific language impairment, $64 \%$ of the children showed evidence of both reading and oral language impairments. Differences were also found between the groups on maths, phonological processing, and spelling measures, with the children displaying both language and reading deficits generally performing at a lower level than the children with specific reading or language deficits. It was concluded that there exist three categories of impairment, children with SLI, children with SRD, and a group of children with both impair- 
ments. As a result, more careful screening needs to be conducted in both clinical and research settings to identify the nature of deficits correctly in children with reading and oral language difficulties.

\section{2: Inhibition of Return in Obsessive-Compulsive Disorder}

Debbie Rankins ${ }^{1,2}$, John Bradshaw ${ }^{2}$, Simon Moss $^{2}$ and Nellie Georgiou-Karistianis ${ }^{2}$

1. Psychology, Murdoch Childrens Research Institute, Parkville, VIC, Australia

2. Psychology, Monash University, Clayton, VIC, Australia

Obsessive-compulsive disorder (OCD) is characterised by repetitive obsessions and/or compulsions that interfere with daily functioning. Neuropsychological studies have suggested that such perseverative behaviours may be due to underlying attentional deficits. Inhibition of return (IOR) is an adaptive mechanism that is thought to assist visual search by biasing attention after a critical, short interval to novel, previously unattended areas. Therefore, this study aimed to examine whether deficient IOR mechanisms could underlie some of the attentional, and perhaps behavioural, problems, reported in OCD patients. Using a computerised IOR paradigm, participants were required to respond to a target that appeared at either the same or different location to a precue that was presented either $100 \mathrm{~ms}$ or $700 \mathrm{~ms}$ earlier. Results indicate that patients had a reduced IOR for targets presented in the left visual field, suggesting lateralised anomalies in shifting attention. Results are consistent with lateralisation anomalies previously reported in OCD.

\section{4: Neuroleptic Malignant Syndrome: A Neuropsychological Case Study}

Dale M. Fogarty

Brain Injury Rehabilitation Unit, Princess Alexandra Hospital, Brisbane, QLD, Australia

The Neuroleptic Malignant Syndrome (NMS) is a distinctive adverse reaction to antipsychotic medications (e.g., phenothiazine, quetiapine) and other agents with dopamine D2-receptor antagonist properties. Characteristic symptoms are severe dyskinesia or akinesia, hyperthermia, blood pressure fluctuations, tachycardia, diaphoresis, dyspnea, dysphagia and urinary incontinence. Although cognitive deficits in the acute stage of the condition are commonly reported to occur and to resolve, the literature is not clear by what metric this conclusion is determined or warranted. Only one brief case study, published in 1986, addresses the neuropsychological sequelae of NMS (Rothke \& Bush, 1986). It identified nonfocalised cognitive dysfunction and persisting memory dysfunction as a salient feature. This case study reports the findings from a comprehensive neuropsychological assessment of a 24-yearold male diagnosed with NMS who had a prolonged akinetic and mute state. His clinical presentation was further complicated by hydrocephalus, ECT and other factors. The testing protocol included the WAIS-III, WMS-III, CVLT-II, Conners CPT, D-KEFS, and other standardised neuropsychological and psychological tests. The results indicated relatively preserved cognitive functioning including attention, working memory and executive functioning. Memory dysfunction (verbal > visual) was a prominent feature of his profile and was significantly below his cognitive ability. Psychomotor speed was mildly reduced and he fatigued easily. These findings suggest that NMS results in specific memory and psychomotor deficits in the post-acute stage of recovery. As these problems have implications both for everyday functioning and for 
guiding rehabilitation interventions, the importance of comprehensive neuropsychological assessment to identify the pattern of intact and disrupted cognitive and memory functions is demonstrated. The long-term residual impairments and their impact still need to be ascertained.

\section{5: Indicators of Neurobiological Deviances in Children with Early Identified Psychopathology}

Bente Gjaerum ${ }^{1}$ and Gudrun B. Gudmundsdottir ${ }^{1}$

1. Research department, Regional Centre for Child and Adolescent Psychiatry, Oslo, Norway

2. Department for Child and Adolescent Psychiatry, Landshospitali, Reykjavik, Iceland

Background: High levels of multi-axial co-morbidity and psychosocial impairment have been found in referred school-aged and younger children. Neurobiological deviances may indicate common causal factors and increase the risk for chronicity in further development. Neurobiological deviances should therefore influence assessment of severity and prognosis and decisions regarding interventions.

Research question: Can neurobiological risk factors be identified from medical history, clinical neurological examination, and supplementary neurological examinations? Sample: 219 children ( $82 \%<7$ years, $66 \%$ boys) referred to educational and psychiatric services had been diagnosed on all axes in ICD-10 (Gjaerum \& Bjornerem 2003). 88 children with IQ $<70$ were excluded due to their high risk for neurobiological disorders.

Method: Available data were analysed in order to identify indicators of neurobiological disturbances. Variables showing abnormality for $30 \%$ or more were analysed further.

Preliminary results: Muscle strength, progressive developmental disorder, hand preference in the family, pregnancy problems, and toilet training were significantly different $(p<.006-.05$ on a Chi Square test of independence) when two groups of children with IQ 70-84 and IQ $=85$ or higher were compared, assuming that the first group more often would show neurobiological abnormality. Play development, psychiatric illness in the child's family, total results on clinical neurological examination, and involuntary movements were close to significance $(p<.07-.08)$. Auditory function, CT, MR, pregnancy problems and social functioning were also significantly different between 5 groups with different psychiatric disorders. These results will be compared to conclusions regarding degree of CNS-disturbance based on neuropsychological assessment.

\section{1: Nonverbal Memory Impairments in Patients with Schizophrenia and Obsessive-Compulsive Disorder}

Myung-Sun Kim ${ }^{1}$, Jun Soo Kwon ${ }^{2}$ and Seung-Suk Kang ${ }^{2}$

1. Psychology, Sungshin Women's University, Seoul, South Korea

2. Psychiatry, Seoul National University College of Medicine, Seoul, South Korea

Tonverbal memory deficits in schizophrenia (SPR) and obsessive-com1 pulsive disorder (OCD) patients were investigated. 13 SPR patients, 8 OCD patients and 13 control subjects participated. Construction accuracy and organisational strategy of Rey-Osterrieth Complex Figure Test (RCFT) were analyzed. Three groups (SPR, OCD and control) showed differences on immediate recall accuracy $(F 2,31=9.307, p<.001)$, and on organisa- 
tional score $(F 2,31=3.770, p<.05)$. SPR and OCD patients showed significantly lower scores on immediate recall and organisation than the controls. The mediating effect of organisational strategy on immediate percent recall (immediate recall score/copy score x 100) was analysed by multiple regression. For OCD and control groups, group had direct effects on mediator and on percent recall, and mediator also had an effect on percent recall. In multiple regression, organisational score still had an effect on percent recall, while group effect on percent recall was reduced and did not reach statistical significance $(p=.434)$. Regression analysis with SPR and control groups showed significant effects of group on percent recall and on mediator accompanied by an effect of mediator on percent recall. However, $\mathrm{F}$ change in multiple regression with the introduction of mediator was not significant $(F$ change $=1.737, n s)$. These results indicate that nonverbal memory impairments in OCD are mediated by organisational deficit, while those in SPR result from the direct effect of group rather than an indirect effect mediated by organisational strategy.

\section{3: Performance of the Neuropsychological Version of Picture Arrangement as a Predictor of Outcome in First-Episode Psychosis}

Gunnar Ganse ${ }^{1}$, Johan Cullberg ${ }^{1}$, Roger Carlsson ${ }^{1}$ and Hakan Nyman ${ }^{1}$

1. Dep of Psychology, Lund university, Lund, Sweden

2. Karolinska Institute, Stockholm, Sweden

3. Uppsala University, Uppsala, Sweden

4. Mental Health, Institute of Public Health, Stockholm, Sweden

Tn the Parachute project 120 patients with first episode psychosis were lexamined by the neuro-psychological version of Wechsler's Adult Intelligence Scale (WAIS-R NI) within two months after admission. The results were compared to those of 30 healthy volunteers, matched in age, educational level and gender distribution. The data of the standard WAIS-R were analysed in relation to BPRS and GAF ratings at baseline, at 1 year and 3 years. Those who had performed low at the initial WAIS-R examination had a less favourable outcome regarding positive and negative but not depressive symptoms or GAF at first year. At the three years follow-up, the initial WAIS$\mathrm{R}$ verbal and performance subscales predicted the level of GAF but not presence of psychotic or depressive symptoms. No other demographic or clinical variables predicted either symptomatic or functional outcome. At the WAIS R NI re-examination, 3 years after admission 68 patients were reassessed and the results show significant improvements in almost all patients. Those patients who had a less favourable outcome at three year had a small or slight improvement in the results of WAIS R. In the NI version of the Picture arrangement subtest, subjects must arrange a set of visual images to perform a correct story sequence, followed by an oral report of the story. Patients with a less favourable outcome made significantly more mistakes in the report of the story, indicating difficulties in aspects of executive functioning. It is suggested that the neuropsychological version of Picture arrangement contributes to the understanding of cognitive functioning in first-episode psychosis as well as prognostic signs Relationships with medication status at examination, differences between diagnostic groups and the effect of the duration of untreated psychosis will be discussed. 


\section{POSTER SESSION 4: CLINICAL ISSUES, TRAUMATIC BRAIN INJURY AND REHABILITATION}

\section{5: Laughter: Neurobiology of an Evolutionary Tool for Social Adaptation}

Sandra C. Rivera-Gutierrez, Mario H. Buenrostro-Jauregui, Nivea A. Chavez-Peraza, Rocio Zavala-Souza and Carlos E. Valencia-Alfonso

Neuroscience Department, Guadalajara University, Guadalajara, Mexico

$\mathrm{T}$ The prevalence of laughter in different cultures, its early presentation in human ontogeny and its stereotypical character are clues that suggest this is a selective process through evolution and is not an eventuality. There are different hypotheses trying to explain what are the evolutionary advantages these processes have, such as increased socialisation, courtship skills, training to combat through playing, signalisation of non-dangerous incongruence clues, and health benefits, among others. Unfortunately, very little is known about the neurobiological mechanisms and evolutionary function of laughter. This review intends to summarise experimental findings concerning the neurobiology of laughter, and its relationship to several evolutionary hypotheses. It is concluded that although there is not a laughter centre in the central nervous system, an important circuit involving the limbic system (hypothalamus and basal ganglia), prefrontal cortex and supplementary motor area seems to exist. Interestingly, these neural structures which are related to laughter are also associated with emotional and social behaviors, and are especially important for the emission and detection of social reinforcement. This reinforcement is very significant in altricial species that develop communities. In humans, both voluntary laughter (cortical control) and involuntary laughter (limbic control) constitute important strategies for social adaptation. Besides, laughing in several daily situations might be explained by the concept of social reinforcement. Therefore, experimental evidence strongly supports the social adaptation hypothesis of laughter. Taken together, the data suggests that this behavior involves biological and social aspects, and its study could be very useful, not only to increase the knowledge of our species, but also to establish technologies that favour it. Finally, further investigations are suggested to study this almost unexplored topic related to some of our best experiences.

\section{9: Coping with Brain-Injury: Belief of Spirit Infestation and Ensorcerement}

Samir Al-Adawi', Atsu A. Dorvlo², Melissa DeVito ${ }^{3}$, Meryl Stein ${ }^{3}$ and David T. Burke ${ }^{3}$

1. Behavioural Sciences, Sultan Qaboos University, Muscat, Oman

2. Department of Mathematics and Statistics, Sultan Qaboos University, Muscat, Oman

3. Department of Physical Medicine and Rehabilitation, Harvard Medical School, Boston, MA

$\mathrm{T}$ The coping mechanisms of Western families when a member sustains traumatic brain injury (TBI) have been well documented in the medical literature; however, there is a paucity of studies that examine other, nonWestern cultures where services for a neuropsychological impairment are rudimentary or do not exist. This paper aims to address this deficiency. In traditional Omani society, sudden personality change with accompanying neurocognitive impairment following a TBI is often attributed to a spirit infestation or ensorcerement. Traditional healing methods, such as exorcism, are often sought in addition to available allopathic medical intervention. This paper reflects on how these beliefs and practices help the family 
cope with the debilitating neuropsychological impairments and personality change that affected a family member due to a traumatic brain injury. It suggests that distress and stress are experienced in a social and cultural context even in the presence of obvious neuropsychological dysfunction precipitated by brain injury.

\title{
80507: Rating The Methodological Quality of Single-case Experimental Designs: The PsycBITETM Scale
}

Robyn L. Tate' ${ }^{\text {, Skye McDonald }{ }^{2} \text {, Leanne } \text { Togher }^{3} \text {, Michael Perdices }}{ }^{4}$ and Anne Moseley'

1. Rehabilitation Studies Unit, Faculty of Medicine, Sydney University, Sydney, Australia

2. School of Psychology, University of New South Wales, Sydney, Australia

3. School of Communication Disorders, Faculty of Health Sciences, University of Sydney, Australia

4. Department of Neurology, Royal North Shore Hospital, Sydney, Australia.

\begin{abstract}
A database, containing published reports of therapies targeting the psychological consequences of acquired brain impairment, is available on PsycBITE $^{\mathrm{TM}}$ (Psychological database of Brain Impairment Treatment Efficacy; www.psycbite.com). PsycBITE ${ }^{\mathrm{TM}}$ is modelled on the Physiotherapy Evidence Database (PEDro) and was developed to aid clinicians to evaluate and implement evidence-based interventions. Reports with empirical data, including systematic reviews, randomised controlled trials (RCTs), nonRCTs, patient series, and single-case experimental designs, are included on PsycBITE ${ }^{\mathrm{TM}}$. A survey of the first 400 reports revealed that the largest proportion (34.5\%) were single-case designs. Like PEDro, a distinctive feature of PsycBITE ${ }^{\mathrm{TM}}$ is the rating of methodological quality. PsycBITE ${ }^{\mathrm{TM}}$ uses the 11-item PEDro scale to rate the quality of group studies (RCTs, nonRCTs, and patient series). The PEDro scale is not appropriate for single-case designs, however, and the PsycBITE ${ }^{\mathrm{TM}}$ scale was therefore developed for this purpose while following the PEDro criteria as closely as possible. Preliminary versions of the scale were piloted on singlecase reports meeting PsycBITE ${ }^{\mathrm{TM}}$ selection criteria by six raters making independent ratings and discussing areas of dissension. As a result a working scale of 11 items was developed. This, like the PEDro scale, has one item for external validity, and 10 focusing on internal validity and statistical analysis. Items include description of patient characteristics, study design, therapy procedures to allow replication, baseline measures, stability of baseline, independence of therapist/assessor, post-treatment/follow-up data, procedures to deal with practice effects, presentation of numerical data and statistical analysis. The application of the PsycBITE ${ }^{\mathrm{TM}}$ scale will thus allow evaluation of the methodological quality of single-case experimental designs, which will be ranked on PsycBITE ${ }^{\mathrm{TM}}$ in order of their methodological strength, enabling end-users to quickly identify trials with greatest internal validity.
\end{abstract}

\section{7: Speed of Information Processing in Persons with Postconcussion Syndrome}

Maria Crawford and Robert Knight

Psychology, Otago University, Dunedin, New Zealand

The aim of the present study was to determine whether access to information is slowed (retrieval-slowing hypothesis) or associations 
between items in memory are lost (structure-loss hypothesis) following head injury. Twenty persons with postconcussion syndrome and 20 controls matched for age, gender, and occupation participated in this study. Participants were given the Controlled Oral Word Association Test (COWAT; Benton \& Hamsher, 1983) and a semantic fluency task where they were asked to name as many fruits and vegetables as possible in 60 seconds. Total scores were calculated and response latencies were measured using a MacLab Chart Sound system. Mean latency, following procedures used by Rohrer, Wixted, Salmon and Butters (1995), was calculated as a test of both the structure loss and retrieval slowing hypotheses. The results failed to find any significant difference in mean latency between the patients and controls. However, the patients produced significantly fewer words and had significantly longer pauses between words than the controls. The patients were also significantly slower at producing their first word on the semantic task and on one trial of the COWAT. Inconsistent with structure loss, the patients produced more words on the fruits and vegetables task than the task requiring them to produce words that were not semantically related. The above findings suggest that retrieval slowing is responsible for the patients' impaired performance on word fluency tasks. Furthermore, response latencies may be a more sensitive measure of the effects of concussion on letter and semantic fluency than the number of words generated.

\title{
71676: Measuring High-Level Mobility Following Traumatic Brain Injury: A Review of Recent Literature
}

\author{
Gavin Williams ${ }^{1}$, Ken Greenwood ${ }^{2}$ and Val Robertson ${ }^{3}$ \\ 1. Physiotherapy, Epworth Rehabilitation, Richmond, VIC, Australia \\ 2. School of Psychological Science, La Trobe University, Bundoora, VIC, Australia \\ 3. School of Physiotherapy, La Trobe University, Bundoora, VIC, Australia
}

The aim of this review was to identify traumatic brain injury studies that reported mobility outcomes and examine which measurement tools they used. The search strategy identified 678 studies. Excluding articles that focused on children, cognitive, behavioural or psychosocial outcomes, 137 studies were collected for full text review. The most frequently used measures for assessing mobility outcomes following brain injury was the Functional Independence Measure (FIM). Findings include the following: activity limitation scales focussing on mobility are seldom used and those that are have a ceiling effect and typically do not measure mobility beyond walking and stair use: inpatient measures such as the FIM are used as outpatient or long-term follow-up measures, applications for which they were not designed; and, "participation" scales are unable to identify if a restriction in participation relates to a mobility limitation. Many studies developed and used their own, non-validated, outcome measures making comparisons and evaluations difficult and some studies did not use any outcome measures at all. A high-level mobility scale is needed to fill the gap between the current "activity" scales that measure mobility to a level of walking and stair use and the "participation" scales that measure leisure and sporting activities. Such a high-level mobility scale is essential to identify and describe the deficits and changes that are currently not measurable following TBI, and may help guide treatment and goal setting for therapists. 


\section{7: Predicting Functional Outcome After Stroke and Traumatic Brain Injury}

Mary Grogan, Suzanne Barker-Collo and Dianne McCarthy

Psychology, University of Auckland, Auckland, New Zealand

Garly assessment after stroke and traumatic brain injury is clinically impor- 1 tant because it provides early identification of deficits and strengths, and can direct appropriate early intensive intervention. Measures that can predict outcome also allow clinicians to focus on specific areas to target for intervention. This study examined the ability of measures of demographic/injury related factors, physical functioning; cognitive measures of memory and attention; and the emotional factors anxiety and depression to predict poststroke and post TBI outcome. 41 participants who had had either a stroke or traumatic brain injury were assessed at admission to a rehabilitation facility and 3 months' post admission. They were asked to complete the Short Form36 (SF-36) at six months' post admission. Results from correlational analysis suggest that age and mood factors (anxiety and depression) at admission and 3 months' post admission were significantly related to SF-36 outcome. Regression analyses showed that age and anxiety scores at 3 months predicted $45 \%$ of the variance in outcome. Currently in NZ $81 \%$ of inpatients do not have access to psychologists and very few inpatients have a mood assessment (Gommans et al., 2003). The results from the current study have implications for the rehabilitation and psychological services offered to inpatients in New Zealand. In addition to physical and occupational rehabilitation, these results point to the need to screen for and target mood disorders.

\section{6: Neuropsychological Functioning Following Severe TBI: A 5 Year Follow-up}

Nigel V. Marsh

School of Psychology, University of New England, Armidale, NSW, Australia

The neuropsychological functioning of a group of 71 adults was assessed at approximately 5 years (mean $=65$ months) following severe traumatic brain injury. The average age of the group was 31 years, and $52(73 \%)$ were male. Overall outcome as rated on the Glasgow Outcome Scale, was $13(18 \%)$ with severe disability, $22(31 \%)$ with moderate disability, and 36 $(51 \%)$ had made a good recovery. Cognitive functioning was assessed by measures of intelligence, attention, verbal and visual memory, and language. Psychosocial functioning was assessed by self-report measures of anxiety and depression, and subjects rated the severity of their current problems on a 22-item measure covering cognitive, emotional, behavioural, and social functioning. The prevalence of difficulties across these domains of neuropsychological functioning, and the interrelationships between them, will be examined.

\section{0: Evaluating an Interactive On-line Cognitive Skill-training Strategy for Persons with TBI}

Wallace Yuen-Leung Soong, Alan Sing-Fai Tam, David Wai-Kong Man and Christina W.Y. Hui-Chan

Rehabilitation Sciences, The Hong Kong Polytechnic University, HungHom, Hong Kong

Objectives: An analogy problem solving approach was hypothesised to enable persons with Traumatic Brain Injury (TBI) to learn better problemsolving skills. Three modes of service delivery were compared, including 
face-to-face, computer-assisted and on-line. Their treatment effectiveness, functionality and usability were evaluated and compared using problems encountered in daily living.

Methods: Sixty Hong Kong Chinese people with TBI were evenly randomly assigned to three 20 -session intervention programs. The programs delivered similar training content via different interaction modes, that is, face-to-face, computer-assisted, and on-line. Training content included basic and functional problem-solving tasks and reflective group sessions. Outcome measures included the Category Test (Halstead Reitan Test Battery); a computerised quiz and a problem-solving self-efficacy scale. The programs were also qualitatively evaluated.

Results: Subjects from all 3 groups generally demonstrated higher self-efficacy and basic problem-solving skills. However, subjects showed differential improvements in different problem-solving skills and self-efficacy. Similar to the other 2 conventional programs, the on-line program also produced significant improvement. This innovative program mode was wellreceived by the subjects though some subjects expressed more real-life simulated practice would improve their functional problem solving skills.

Conclusions: Persons with TBI can improve their daily problem solving through systematic and successful environmental encounters. The on-line program is an effective option for those receiving treatment within their living environment. However, the program effects on generalisation and maintenance remain to be determined.

\title{
73103: Selective Short-Term Memory Impairment Following Closed Head Injury: Possible Role of the Central Executive
}

\author{
Ashok Jansari', Samantha Cole ${ }^{1}$ and Rosaleen McCarthy ${ }^{2}$ \\ 1. School of Psychology, University of East London, London, United Kingdom \\ 2. Department of Psychology, University of Cambridge, Cambridge, United Kingdom
}

Selective impairments in short-term memory tend to be more rare than reports of long-term memory problems. Single case studies, however, have shown that patients can have selective impairments in just verbal shortterm memory; for example Shallice \& Warrington (1970) reported the case of KF who had a digit span of 2, which has been interpreted as a deficit in the Phonological Store of Baddeley \& Hitch's (1974) Working Memory model. We report the case of a patient with a selective short-term memory problem following closed head injury but who, unlike KF, has an intact digit span and is unimpaired on other simple measures of working memory function. Following his reports of particular problems when trying to articulate complex sentences when stressed and difficulty following conversations when more than one person is speaking, a detailed case study is presented whereby potential problems at the resource level of the Central Executive's attentional system are investigated. The bearings that the findings have on further understanding of short-term memorial processes are discussed. 


\title{
73116: Composite Assessment of TBI Patients: SMS Simulations
}

\author{
Usha Satish and Siegfried Streufert
}

Psychiatry, SUNY Upstate Medical University, Syracuse, NY, USA

\begin{abstract}
Simulation data were obtained from patients with a history of closed head

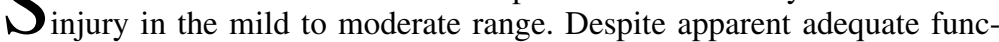
tioning on standard tests, many patients demonstrated considerable deficits in real world relevant tasks. The SMS simulation provides the opportunity to specifically delineate these deficits and study the impact of various treatment options. Patients who had experienced prior brain trauma were, on the average, less active, responded inadequately to information from the environment, showed little initiative, were dependent on external contextual cues (seeking more information to use as a basis for action, yet did not utilise that information effectively), responded poorly to an emergency, and showed little breadth. They showed marginal planning ability and displayed limited use of strategy, except in highly externally cued (contextual) situations. In general, their behaviors reflected a restricted orientation toward situational demands with a primary focus on one (or very few) task components. As a result, their scores on "contextual" performance measures and on information search activity tended to be relatively high. In contrast, their understanding of purposes or goals as well as their effectiveness in handling more complex real-world task demands was absent or limited.
\end{abstract}

\section{3: Risk of Unemployment Following Traumatic Brain Injury}

Sureyya Dikmen ${ }^{1,2}$, Jason Doctor ${ }^{4,1}$, Jon Castro ${ }^{4}$, Robert Fraser ${ }^{5,1}$, Joan Machamer ${ }^{1}$ and Nancy Temkin ${ }^{2,3}$
1. Rehabilitation Medicine, University of Washington, Seattle, WA
2. Neurological Surgery, University of Washington, Seattle, WA
3. Biostatistics, University of Washington, Seattle, WA
4. Medical Education, University of Washington, Seattle, WA
5. Neurology, University of Washington, Seattle, WA

Objective: Examine the risk of unemployment 1 year after traumatic brain injury (TBI) relative to that expected in the general population.

Design: Inception cohort longitudinal study of TBI cases. General population risk from the US Current Population Survey.

Setting: Level I trauma centre.

Patients: 420 consecutive hospital admissions of adults with traumatic brain injury who were working at the time of injury.

Main Outcome: Work status at 1 year.

Results: $42 \%$ of TBI cases were unemployed vs. $9 \%$ expected, risk ratio $(\mathrm{RR})=4.5$. The RR for unemployment was higher in males, those with higher education, more severe injuries, and more impaired early neuropsychological or functional status. The difference in unemployment rates gave similar results for gender, severity of injury and early neuropsychological and functional status. However, for education, the actual difference was smaller among those more educated, but the rate in the general population was smaller, yielding a higher risk ratio. Those with under a high school education had $54 \%$ unemployment after TBI vs. $14 \%$ expected (difference 
$=40 \%, \mathrm{RR}=3.8$ ) while college graduates had $24 \%$ unemployed after TBI vs. $4 \%$ expected (difference $=20 \%, \mathrm{RR}=5.6$ ).

Conclusions: After correcting for population risk for unemployment, unemployment is substantially higher after TBI for people who were employed when they were injured. The excess varies depending both on the characteristics of the injury and of the person injured. For characteristics associated with rates of unemployment in the general population such as education, risk ratio and excess percent unemployed give different views of what groups are more severely affected.

\title{
73125: An Epidemiological Investigation of Head Injury Presentations over 6 Years in a Local UK Health District Population
}

\author{
Philip J. Yates ${ }^{1,2}$, Becky Jenkins ${ }^{4}$, Lyn Ellett ${ }^{1}$, Adrian Harris ${ }^{3}$ and Huw Williams ${ }^{2}$ \\ 1. Clinical Psychology, Mardon Neuro-rehabilitation Centre, Exeter, United Kingdom \\ 2. School of Psychology, University of Exeter, Exeter, United Kingdom \\ 3. Accident \& Emergency, Royal Devon \& Exeter Hospital, Exeter, United Kingdom \\ 14. Public Health, Peninsula Medical School, Exeter, United Kingdom
}

\begin{abstract}
A descriptive analysis was carried out of data collected over six years on up to 8000 head injury presentations at an Accident \& Emergency Department serving a local health population of approximately 350,000. Too few UK studies on the epidemiology of head injury exist to make for meaningful interpretation at a local level. The literature does however suggest that significant local and regional variation in incidence can occur, and that service provision should reflect this. This paper describes two phases of analysis undertaken in order to derive a local incidence rate. Initial trends were identified according to severity, sex, age, area of residence, and case follow-up. Significant variation was found on these factors. A provisional estimate of annual incidence was calculated at 361 per 100,000 (335/100,000 for minor cases; 26.3/100,000 for moderate-severe cases). A second phase of analyses was carried out on the data to refine these estimates. This will be presented in detail and includes measures of social deprivation to further understand the nature of case presentations. The implications of the findings for clinical neuropsychological services will be considered.
\end{abstract}

\section{9: Apathy and Affective Functioning in Cross-Cultural Survivors of Traumatic Brain Injury}

Samir Al-Adawi ${ }^{1,3}$, Atsu S. Dorvlo², Loyal Jacob³, David T. Burke ${ }^{3}$, Charles C. Huynh ${ }^{3}$ and A Al- Hussaini ${ }^{1}$

1. Department of Behavioural Science, Sultan Qaboos University, Muscat, Oman

2. Department of Mathematics and Statistics, Sultan Qaboos University, Muscat, Oman

3. Department of Physical Medicine and Rehabilitation, Harvard Medical School, Boston, MA, USA

isturbance of motivation and its relationship to depression continues to spark contradictory findings among European and North American populations. Could a cross-cultural study shed some light on the situation? This study aims to detect the prevalence of apathy and to test whether the Apathy Evaluation Scale (AES) can detect the presence of depression in survivors of traumatic brain injury (TBI) in Oman. Eighty subjects who sustained a TBI were given an Arabic version of the AES and were also interviewed with the semi-structured Composite International Diagnostic Interview (CIDI). The authors found that, based on CIDI, the incidence of 
apathy and depression in the TBI in Oman is similar to that reported elsewhere. However the AES has poor discriminatory power in identifying cases of depression in this population. Could this imply that the AES is not culture sensitive? The results of this study emphasise the importance of developing assessment tools that are culturally sensitive in the light of the rising incidence of TBI in developing countries such as Oman.

\section{6: Valproate for Altering Mood after Traumatic Brain Injury}

Nancy Temkin ${ }^{1,2}$, Joan Machamer ${ }^{3}$, Jesse Fann ${ }^{4}$, Charles Bombardier ${ }^{3}$ and Sureyya Dikmen ${ }^{3,1}$

1. Neurological Surgery, University of Washington, Seattle, WA

2. Biostatistics, University of Washington, Seattle, WA

3. Rehabilitation Medicine, University of Washington, Seattle, WA

4. Psychiatry and Behavioral Sciences, University of Washington, Seattle, WA

Wood disorders such as irritability, depression, and anxiety are commonly reported after traumatic brain injury. Valproate is frequently used as a mood stabilising drug.

Objective: To determine whether valproate has any effect on mood in those with traumatic brain injury.

Design: Randomised, double-blind, clinical trial to compare the effects of valproate and phenytoin for seizure prophylaxis. Assessments were done during valproate treatment at 1 and 6 months after injury.

Setting: Level I trauma centre.

Patients: 189 patients on their assigned, blinded drug tested at 1 month and 145 at 6 months. Participants were at least 14 years old and had complicated mild to severe injuries.

Interventions: 1 week of phenytoin (followed by placebo) or 1 month or 6 months of valproate.

Main outcome measures: Brief Symptom Inventory (BSI).

Results: Despite depressive symptoms being quite common, there was no effect of valproate on depressive symptoms reported $(37 \%>1$ sd above on BSI depression at both 1 and 6 months, $p=.84$ and .44 comparing groups). BSI hostility scale reflecting irritability indicated somewhat fewer problems overall, but again no treatment effect (about 25\% >1SD, $p=.72$ and .79). Results on anxiety are less clear. Cross-sectionally, there was no treatment effect ( $p=.78$ and .84 ), but an analysis of change from 1 to 6 months suggested a positive impact of valproate on anxiety $(p=.04)$.

Conclusions: Despite some elevations, there is no indication valproate decreases depression or irritability. There is a suggestion of a positive effect of long-term valproate use on anxiety. 


\title{
73190: Pragmatic Communication Skills in Children Following Traumatic Brain Injury
}

Simone Bassi ${ }^{1}$, Alan Tucker ${ }^{1}$, Vicki Anderson ${ }^{2,3}$, Sue Morse $^{5}$, Flora Haritou ${ }^{5}$, Cathy Catroppa ${ }^{3,6}$ and Jeffrey Rosenfeld ${ }^{4}$
1. Psychology, Victoria University, Melbourne, VIC, Australia
2. Psychology, University of Melbourne, Melbourne, VIC, Australia
3. Psychology, Royal Children's Hospital, Melbourne, VIC, Australia
4. Neurosurgery, Royal Children's Hospital, Melbourne, VIC, Australia
5. Speech Pathology, Royal Children's Hospital, Melbourne, VIC, Australia
6. Murdoch Children's Research Institute, Melbourne, VIC, Australia

\begin{abstract}
$\mathrm{T}$ Traumatic brain injury (TBI) is one of the most common causes of acquired disability during childhood. Most of the literature to date has used standardised tests to explore the impact of TBI on children's cognitive skills, with little exploration of children's higher level functional skills, especially in the language/communication domain. Difficulties in these areas are likely to contribute to persisting social and academic difficulties often associated with TBI. The aim of the present study was to explore the impact of TBI on children's pragmatic communication skills, their ability to effectively use language in a social context. A longitudinal design was used to compare the pragmatic communication skills of children who had sustained mild $(n=10)$ and moderate-severe $(n=14)$ TBI between the ages of 3 and 7 years to a group of uninjured children $(n=12)$. The children were matched on age, gender, Full Scale IQ and pre-injury Vineland Adaptive Behavior Score. Children were asked to explain how to play a popular children's game initially after injury, with follow-ups at 6 and 30 months. Explanations were rated on the quantity and quality of information supplied as well as the organisation and efficiency of responses. Results are discussed in terms of the development of executive skills and the heterogeneity in outcome following childhood TBI.
\end{abstract}

\section{4: Management of Posttraumatic Epilepsy in People with Brain Injury: A Psychoeducational Perspective}

\section{Michelle L. Bellon}

Disability Studies, Flinders University, Adelaide, SA, Australia

Aim: This study identifies the behaviours of 18 adults with posttraumatic epilepsy (PTE), and examines the effects of a psychoeducational intervention on participants' personal management and lifestyle, incidence of seizures, as well as measured psychosocial and cognitive functioning.

Method: Participants were assigned to the intervention group through selfselection $(n=9)$, with a matched no-treatment control group established for valid comparison $(n=9)$. The intervention involved 6 months of weekly 2hour workshops plus counselling, addressing PTE management. Data was collected from medical records, interviews, participant observation, seizure diaries and questionnaires (Washington Psychosocial Seizure Inventory, Stigma Scale, Perceived Wellness Survey and San Diego Questionnaire), completed independently by participants and their nominated family member/friend. Data collection occurred at baseline and post-intervention, forming a repeated measures design. A third round of data collection will be conducted at a 6-month follow-up. 
Results: Discrepancies between participant and significant other's perceptions reduced following the intervention, indicating an improvement in selfawareness by participants. Results demonstrate the effects of isolation, lack of understanding and support, and influence of structured networks in facilitating effective management of PTE. Discussion: PTE is a serious complication of acquired brain injury, affecting long-term rehabilitation outcome, and educational, vocational and personal achievement. Skill development and effective management of PTE requires careful nurturing of participants allied to sensitivity of their needs. This builds self-awareness and confidence such that seizures are better managed, participation in community activities is established, and the rehabilitation process is enhanced. This research was conducted during the tenure of a research scholarship from the Epilepsy Association, Australia.

\title{
73241: Efficacy of Computer-assisted Memory Rehabilitation Program for Persons with Traumatic Brain Injury: Development and Evaluation
}

\author{
David W. Man, Zu Lin Dou, Sing Fai Tam and Christina W.Y. Hui-Chan \\ Rehabilitation Sciences, The Hong Kong Polytechnic University, Hong Kong, Hong Kong
}

Background: Efficacy of computer-assisted cognitive rehabilitation (CACR) for persons with traumatic brain injury (TBI) is increasingly being recognised. The present randomised clinical trial examined a new CACR strategy in treating persistent short-term memory (working memory) deficits. The strategy adopted an eclectic approach that integrated errorless learning and multi-sensory environment enrichment concepts.

Method: Sixty persons with TBI were recruited from a treatment programme and randomly divided into three groups: CACR group underwent 20 one-hour computerised remediation sessions, a second group (nonCACR) underwent a time-matched face-to-face therapist-led memory training task, and a third control group received no research intervention (only presented with non-specific cognitively demanding tasks). They all completed the various treatment conditions as well as a battery of computerised and neuropsychological tests (e.g., RBMT) designed for longitudinal measurement of changes in memory and outcome measures.

Results: Initial findings indicated that both CACR and non-CACR had significant improvement in memory following treatment and 1-month followup. Though the results did not support differential efficacy between CACR and the conventional programme in augmenting the memory process, feasibility of the CACR was evidenced. Methodological considerations of cognitive remediation paradigms will also be discussed.

\section{5: Neuroimmune Processes and Categories of Performance Variability Within or Across Sessions after Mild Head Trauma (MHT)}

F.F. LeFever ${ }^{1}$ and John Hasenkam ${ }^{2}$

\section{NYNG, New York, NY; 2. Bahrs Scrub, Nobby Beach, QLD, Australia}

Tntrasubject variability of cognitive performance within or across sessions 1 is common in brain trauma. In mild head trauma (MHT), evidence of structural brain damage may be lacking or ambiguous, as may be objective evidence of significant cognitive deficits; but in variability across time, MHT patients resemble those with moderate-severe TBI. Neuroimmune responses to experimental MHT resemble those in a wide variety of brain 
insults (ischemia, seizures, infection, etc.) and provide a basis for several types of long-term alterations in specific basal or phasic neuroimmune activities with potential for causing chronic but fluctuating dysfunction. Fluctuations have been categorised according to modulating conditions and time course: (1) brief and task-specific ("mental fatigue" with lapses of seconds or minutes; LeFever, 1996); (2) longer and not necessarily task- or situation-specific (precipitated by somatic as well as emotional stress, lasting a day or so; LeFever, Society for Neuroscience 1998); (3) much longer, unrelated to task or situation (days or weeks, due to IL-1beta response to systemic or local non-brain infection; e.g., Kelley, Bluthe, Dantzer et al., 2003). To these, we add sleep disturbance and/or desynchronisation among various independent but normally coordinated circadian rhythmic processes. Neuroimmune sequelae involving upregulation or disordered production of cytokines has been shown to influence circadian mechanisms. Although they have been shown to have direct effects on synaptic activity and on cognitive functions, if they impair synchronisation of separate systems which normally have parallel circadian rhythms at levels which in themselves might have relatively minor direct impacts on neural/cognitive function, there are several possibilities for sporadic or periodic effects on mood, overt behaviour, or cognitive functions (e.g., asynchrony of peak overt activity and peak slow-wave power in the EEG; Roky et al. 1999).

\section{9: Barriers to Driving after Traumatic Brain Injury}

Lisa J. Rapport ${ }^{1,3}$, Robin A. Hanks ${ }^{2,3}$, Renee D. Coleman ${ }^{2,3}$ and Carole Koviak ${ }^{2}$

1. Psychology, Wayne State University, Detroit, MI, USA

2. Psychology and Neuropsychology, Rehabilitation Institute of Michigan, Detroit, MI, USA

3. Physical Medicine and Rehabilitation, Wayne State University School of Medicine, Detroit, MI, USA

riving status and perceptions of barriers to the resumption of driving after traumatic brain injury (TBI) were examined among 51 TBI survivors. Perceptions of barriers to driving provided unique information in predicting subjective and objective indices of community integration, such as sense of belonging, social mobility, and occupational integration, even after accounting for social support, negative affectivity, and use of alternative transportation. Moreover, survivors who had not resumed driving showed poorer community integration than those who had resumed driving. Survivors' perceptions of their barriers to driving accounted for substantial variance in driving status. Physical, cognitive, psychological, and resourceoriented barriers were modestly related to driving outcome; however, social barriers such as directives against driving from significant others accounted for the most variance in survivor driving status. Drivers and non-drivers did not differ in perceptions of general social support, or in levels of positive or negative affectivity. Although general perceived social support and negative affectivity converged with subjective indices of community integration, they were unrelated to both perceptions of social barriers to driving and driving outcome. Thus, survivors distinguished between social support, which was generally perceived as adequate by this sample, and social barriers imposed by the same network that hindered their resumption of driving. The findings support prior research indicating that significant others have substantial influence in post-TBI driving outcome and highlight the importance of independent driving to community integration. 


\section{3: Behaviours Suggestive of Frontal-Subcortical Dysfunction 1-6 Years after Traumatic Brain Injury: A Comparison of Patient Versus Knowledgeable Informant Report}

Laura Seibert ${ }^{1,2}$ and Robert K. Heaton ${ }^{1,2}$

1. Department of Psychiatry, University of California, San Diego, San Diego, CA, USA

2. Department of Psychology, San Diego State University, San Diego, CA, USA

B ehavioural changes associated with disturbance to frontal-subcortical circuitry are frequently reported sequelae of traumatic brain injury (TBI). The Frontal Systems Behaviour Scale (FrSBe), a behavioural rating scale with demonstrated validity to assess these changes, was administered to 27 individuals 1-6 years post TBI and independently to knowledgeable informants (family member/significant other). The FrSBe assesses the frequency of behaviours in three domains: apathy (e.g., initiation difficulties, task impersistence); disinhibition (e.g., irritability, emotional lability), and executive dysfunction (e.g., perseveration, trouble multi-tasking). As groups, the reports of individuals with TBI and informants indicated comparable rates of clinically significant behavioural problems after injury, for each domain (per TBI-affected individuals: 30\% showed signs of apathy, $37 \%$ disinhibition, and 33\% executive dysfunction; per informants, 33\%, $41 \%$, and $26 \%$, respectively) and overall (per TBI-affected individuals, $37 \%$; per informants, $33 \%$ ). Both groups reported more signs of apathy, disinhibition, and executive dysfunction after affected-individuals injury as compared to before. These group results are qualified, however, by the finding of no significant correlations between the ratings of TBI-affected individuals and the ratings of informants for any domain and overall. The two groups disagreed on the classification of TBI-affected individuals as clinically impaired overall or not after injury for 11 of the $27(41 \%)$ affected individuals. The two groups were also in disagreement on the classification of TBI-affected individuals as clinically impaired overall or not before injury, for 6 of the 27 (22\%) affected individuals. The reasons for the poor concordance between the reports of TBI-affected individuals and those of knowledgeable informants is an area in need of further investigation.

\section{1: Using Rasch Statistics to Rescale Neuropsychological Performance in Patients with Postconcussion Symptoms}

\section{Raymond C. Chan ${ }^{1,2}$, John M. Linacre ${ }^{3}$ and Benjamin D. Wright ${ }^{3}$}

1. Psychology, Sun Yat-Sen University, Guangzhou, China

2. Psychiatry, The University of Hong Kong, Hong Kong, Hong Kong

3. MESA Lab, Education, University of Chicago, Chicago, IL

Tn analysing clinical or educational data, subjects or patients may already Ibe characterised with a specific set of "numbers" that are asserted to be linear measures that could be compared with one another directly. However, it is not clear that what is the linear transformation between the measure and logits. It is not even known what the specific "number" in one neuropsychological test tells you in comparing with the exact "number" in another test of similar measure. The traditional approach of using the standardised z-score or T-score within a specific sample, however, limits to single inventory. In clinical practice, however, psychological and neuropsychological tests are usually assessed with a wide range of measures on the same construct. This approach thus still overlooks the direct comparison of measures 
of different inventories. This study aims to take the common metric into consideration and to optimise direct comparison of neuropsychological measures in a group of patients with traumatic brain injury using Rasch Model Analysis. In this technique, one first transforms the different performance scores to a "logits" metric. Then, each performance score is transformed to a logit scale of "difficulty" along which different levels of difficulty could be directly compared with one another. In doing so, a common profile score can be established to locate the subject's performance within the set of neuropsychological measures.

\section{4: Evidence-based Assessment of Brain Dysfunction in Head Injury}

Kathy Conne/R,1, Evian Gordon ${ }^{2,1}$, Kamran Fallahpour ${ }^{5,1}$, Carolyn Davis ${ }^{2,1}$ and Richard Clark ${ }^{7,1}$
1. International Brain Database, Brain Resource Company, Sydney, NSW, Australia
2. International Brain Database, Brain Resource Company, Sydney, NSW, Australia
3. Brain Resource Company, Brain Resource Company, Sydney, NSW, Australia
4. International Brain Database, Brain Resource Company, Sydney, NSW, Australia
5. Institute of Neuropsychology \& Cognitive Performance, Brain Resource Company, New York City, NY, USA
6. Brain Resource Company, Brain Resource Company, Sydney, NSW, Australia
7. Social Science, Flinders University, Adelaide, SA, Australia

$\mathrm{T}$ This study serves to elucidate evidence-based changes in electrical brain function and psychometrics, in patients with a head injury referred for Medico-Legal evaluation. The subject group consists of 7 patients with a history of head trauma (age range 34-52). The normal control group was age matched and drawn from the Brain Resource Standardised International Brain Database. Measures of electrical function (EEG eyes closed, auditory oddball and working memory paradigms), and a battery of psychometrics (including assessment of sensory-motor-spatial, memory, attention, language and executive functions) were evaluated. MANOVA and ANOVA analysis of the data showed significant differences in EEG power, amplitude of the late component oddball and working memory event related potentials (ERPs) - which highlighted significant disturbances in context processing (P300). The pattern of psychometric disturbances highlighted significant differences in all five core domains of cognitive functioning. Insights of the inter-relationships between psychophysiological and psychometric data, from over 1000 normal subjects in the International Brain Database, were used as a frame of reference to interpret the potential functional significance of these findings. The frame of reference also allowed individualised patterns of dysfunction to be elucidated, with respect to the controls in the International Database.

\section{5: Environmentally Induced Transient Cognitive Improvements in Healthy Subjects: Implications for Neurorehabilitation Following Brain Injury}

Robin Green ${ }^{1,2}$, Brenda Melo', Le-Anh Ngo ${ }^{2}$ and Catherine Skene ${ }^{2}$

1. Toronto Rehabilitation Institute, Toronto, ON, Canada

2. Graduate Department of Rehabilitation Science, University of Toronto, Toronto, ON, Canada

$\mathrm{O}$ ur program of research examines whether it is possible to measure (1) the transient improvement in cognitive functioning that people anecdotally report after periods of intensive mental activity (e.g., studying for exams), and (2) subsequent regression to pre-intensive activity levels of 
cognitive functioning. If such transient improvements occur, this would have significant implications for neurorehabilitation following brain injury. The stimulating neurorehabilitation environment may promote transient neuroplastic changes; upon discharge from the environment, transient improvements may dissipate. Preliminary findings from our traumatic brain injury recovery study reveal that some patients do indeed decline after discharge from neurorehabilitation. We present here the results of our first experiment: Can performance of normal controls be enhanced by intensive cognitive stimulation? Ten normal controls were administered memory, attention and speed of processing tests before and after a two-week intervention, using alternate forms to minimise practice effects. The intervention entailed daily memorisation of prose passages and speeded mental calculations (intended to tap effortful processing). Sixteen control subjects performed the pre- and post-assessments, without the intervention. Both groups improved significantly on speed of processing tests, suggesting practice effects; however, only the intervention group showed significant improvements on memory and attention tests. Consistent with anecdotal report, results suggest it is possible to transiently enhance cognitive functioning in normal controls through stimulation. Follow-up assessment will be undertaken to provide further confirmation that cognitive improvements were transient. If intensive stimulation confers transient benefits, then post-discharge, ongoing cognitive stimulation may be necessary to maintain rehabilitation benefits.

\title{
POSTER SESSION 5: LANGUAGE, SEMANTIC MEMORY, MEDICAL CONDITIONS
}

\section{6: The Presurgical Evaluation of Language Localisation and Lateralisation Using fMRI}

\author{
Sarah Tomaszewski Farias and Gregory Harrington
}

Neurology, University of California, Davis, Sacramento, CA, USA

The intracarotid amobarbital procedure (IPA), or Wada test, is currently the gold standard in determining language lateralisation in the presurgical assessment of epilepsy and other CNS lesions. Functional MRI is fast approaching as a viable alternative with good concordance between the two methods. Additional benefits of fMRI include intra-hemispheric localisation of specific language abilities. However, fMRI has yet to achieve its full potential as a clinical tool in this regard. In this study we present data on the development of a comprehensive language lateralisation and localisation fMRI protocol in a sample of 38 right-handed controls. Language paradigms examined included: confrontation naming, verb generation, auditory and visual sentence reading, semantic decision-making, and story listening. We compared the laterality index (LI) for each task across anterior and posterior language zones to examine: (1) how laterality indices differ for each paradigm across cortical regions of interest, and (2) which groups of paradigms would produce activation in both regions of interest in $100 \%$ of subjects. Results showed that LIs varied considerably between tasks and were heavily dependent on the region of interest. Some of the paradigms that had a stronger receptive component produced stronger LIs for the posterior language zone. Verb generation was the single best task, both in terms of generating activation in a high percent of subjects, and in producing high LIs across both cortical regions. Various combinations of tasks are pre- 
sented which provided activation in $100 \%$ of the sample and produce strong activation in both anterior and posterior language zones.

\title{
73413: Neural Correlates of Semantic Drawing: An fMRI Study
}

\author{
Greg S. Harrington ${ }^{1}$, Dana Farias ${ }^{2}$ and Chris Davis ${ }^{2}$ \\ 1. Radiology, UC Davis Medical Centre, Sacramento, CA, USA \\ 2. Physical Medicine and Rehab, UC Davis Medical Centre, Sacramento, CA, USA
}

\begin{abstract}
$\mathrm{D}$ rawing familiar objects is a complex motor skill with high visuospatial demands that requires access to semantic memory. However, there have been few neuroimaging studies investigating the neural substrates for drawing. Drawing can be used to communicate non-verbally and has been utilised in the clinical setting with aphasic patients to help facilitate the naming of objects. Farias et al. (2003) compared the mental imagery of drawing versus writing and found similar activation patterns for the two tasks with stronger activations for drawing in regions involved in visuospatial and language processing. The goal of the present study was to investigate the semantic aspects of drawing objects by comparing the drawing of familiar objects versus unfamiliar objects using simulated drawing. For the fMRI experiment, the subjects $(n=9)$ simulated (imagined) drawing either a familiar object or an unfamiliar object that they previously viewed on a screen. The two conditions were compared with a general linear test. There were multiple areas in the left hemisphere that were more active for drawing familiar objects compared to unfamiliar objects. These areas include the inferior posterior temporal (BA37), anterior inferior frontal (BA 46), anterior cingulate, precentral, inferior parietal and supplemental motor areas. We hypothesise that the BA 46 activation is due to the selection of specific semantic features of the object as well as retrieval of information regarding the perceptual input of the object versus the stored memory. This activation is linked to BA 37, which is involved in object recognition as well as semantically mediated speech.
\end{abstract}

\section{9: Brain Process on Single Tool Use}

Yoshitsugu Nakagawa', Mika Otsuki ${ }^{2}$ and Maki Inokawa ${ }^{3}$

1. School of Nursing and Social Services, Health Sciences of Hokkaido, Tobetsu, Japan

2. School of Psychological Science, Health Sciences of Hokkaido, Sapporo, Japan

3. Depart. Rehabilitation, Kyoritsu Rehabilitation Hospital, Kawanishi, Japan

The underlying mechanism of apraxia on single tool use is still on debate. To specify which kind of tool cannot be used, we theoretically classified tools into familiar and unfamiliar tools. Then, to specify which kind of process for performing single tool use can be damaged, they were divided into two distinctive phases: (1) a phase for evoking or organising usage of familiar or unfamiliar tools; (2) a phase for realising movements according to the evoked or organised usage. Utilising these theoretical distinctions, we investigated two patients, showing deficits on process for single tool use following left hemisphere damage. As a result, the underlying mechanisms for apraxia on single tool use (ideational apraxia) can be attributed to a deficiency in the process for evoking movements for using familiar tools. In addition, "familiar" tools should be actually restricted to the "tools supported by repetitive or symbolic movements". Manipulation of the tools supported by these repetitive or symbolic movements could facilitate action-somatosensory feedback linkage (i.e., skill). Thus, failure of evoking 
this linkage may result in apraxia of single tool use (ideational apraxia). Use of other tools lacking this linkage may be mediated by another strategy.

\title{
73343: Preliminary Standardisation of the Minnesota Test for Differential Diagnosis of Adult Aphasia in Greek Population
}

\author{
Tafiadis C. Dennis and Konstantinia Arampatzi
}

Speech Therapy, Intistute of Technology Patras, Agrinio, Greece

Durpose of the present study was the preliminary standardisation and validation of an adult aphasia assessment battery for the Greek population. The Minnesota test for the differential diagnosis of aphasia (MTDDA) was originally created by Hildred Schuell in 1946 and was later revised in 1972 by Jenkins, Jimenes-Pabon, Shaw and Sefer (1975). The battery is used as a diagnostic scale for the differential diagnosis of adult aphasia and measures the language skills in the aphasic population. The battery was administered to 30 aphasic participants and 50 non-aphasic participants recruited from Greek health settings, aged 19-81 years. Statistical analysis of the data revealed that the results obtained are generally consistent with the results reported in other countries. No statistically significant differences were found between the results obtained for the Greek population and the results reported in the U.S.A. population in all 5 diagnostic categories (auditory disturbance, visual and reading disturbance, speech and language disturbance, visuomotor and writing disturbance, disturbance of numerical relations). The battery appears to be sensitive to adult aphasic symptomatology in the Greek population and presents satisfactory criterion and content validity as the aphasic participants assessed demonstrated clear patterns of deficit. The usefulness of the battery for the Greek population in clinical and research settings is also discussed.

\section{3: Frontal Pure Agraphia:}

\section{The Mechanism of Writing Related to the Frontal Lobe}

Makiko Rai', Mika Otsuki², Buhan Hyon'1, Hiroshi Moriwaki ${ }^{1}$ and Hiroaki Naritomi ${ }^{1}$

1. Department of Cerebrovascular Medicine, National Cardiovascular Center, Osaka, Japan

2. School of Psychological Sciences, Health Sciences University of Hokkaido, Hokkaido, Japan

Background and Purpose: Frontal lobe lesions can cause pure agraphia, although the mechanism of writing disturbance remains unknown. We evaluated patients with frontal agraphia to clarify the issue.

Methods: We examined three Japanese patients who developed writing impairment after brain infarction limited to the frontal lobe. All lesions were assessed by MRI. Western Aphasia Battery and other cognitive tests were administered within one month after onset. We assessed writing errors by writing single letters, single words, several words, and sentences, using kana (syllabograms) and elementary school levels of kanji (morphograms).

Results: All patients presented pure agraphia. Their common lesions were located in the precentral gyrus of the dominant hemisphere. They wrote wellformed letters and all single kana letters correctly. However, they made $1.3-16.6 \%$ errors in writing single words. There was no significant difference in frequency of errors between kana and kanji. The most frequent errors were paragraphia and no response. They tended to make more mistakes when they wrote longer words or sentences. Paragraphic errors were frequently observed 
with spontaneous writing. In writing kana words, the more letters were comprised of the words, the more paragraphic errors resulted.

Conclusions: Two points are considered to be impaired in the writing processes. The first is evoking the visual image of letters and the unit of letters which consists of words. The second is selection and arrangement of the appropriate letters for writing words and sentences. The latter one appears to be more severely impaired in frontal pure agraphia.

\title{
72508: Treatment of Acquired Dysgraphia: Selective Benefit from Visual Mnemonics
}

\author{
Laura Schmalzl and Lyndsey Nickels \\ Macquarie Centre for Cognitive Science, Sydney, NSW, Australia
}

Tn contrast to the numerous treatment studies of spoken language deficits, there have been relatively few studies concerned with the treatment of spelling disorders. Among these, there have been only a small number that have targeted specific components of the spelling process. We describe a successful single case treatment study for FME, a woman with acquired dysgraphia, which was conducted within a cognitive neuropsychological framework. Pre treatment assessment revealed a semantic deficit, impaired access to output orthography and probable additional degradation of the actual representations within the orthographic output lexicon. The treatment study was therefore directed toward relearning spellings by strengthening, and facilitating access to, specific orthographic representations for writing. In order to maximise the functional outcome for FME, treatment was focused on high frequency irregular words. The treatment program was carried out in two phases, one without and one with the use of mnemonics, and the results showed a selective training effect with the mnemonics alone. Treatment benefits were item specific but long lasting, and a significant improvement in FME's spelling performance was still evident at 2 months post treatment. The current study confirms how cognitive neuropsychological theories and methods can be successfully applied to the assessment of acquired spelling impairments, and exemplifies how treatment with carefully designed mnemonics is of benefit if the inability to retrieve orthographic representations for writing is aggravated by a semantic deficit.

\section{3: Brain Mechanisms of the Readiness to Speak}

Janna M. Glozman, Alexander V. Vartanov and Andrey A. Kiselnikov

Psychology, Moscow State University, Moscow, Russian Federation

$\mathrm{T}$

The readiness to speak is an important component of the verbal functional system. Component disturbances form the main mechanism of stuttering. This paper is a cross-disciplinary cognitive and neural analysis of the mechanisms of speech in normal and stuttering subjects. It is aimed to identify the neural and neuropsychological mechanisms of stuttering, a frequent and stable disorder of speech, revealed in about $3.5 \%$ of the population, both children and adults, severely interfering with their cognitive and social functioning. The original method of analysis of electrical brain waves images preceding pronunciation combining: the 16 channel electroencephalography (EEG) with spectral analysis; event related brain potentials with multimodal approach to source reconstruction; the dipoles model of space localisation of cerebral activation with differentiation of cortical and subcortical components 
of this activation; mapping methods - together with the magnetic resonance imaging (MRI) and the comprehensive neuropsychological assessment using Luria methods reveal the neural mechanisms of readiness for speech (verbal action preparation). The preliminary cerebral activity is synchronised both with verbal sounds perception and with word pronunciation. A comprehensive neuropsychological assessment proves that the neural organisation of the readiness to speak is related to individual patterns of cognitive functioning and the cerebral dominance of subjects. Both inter- and intrahemispheric interactions of brain structures realise the readiness potentials. A longitudinal follow-up proves that these interactions are the subject of changes during speech rehabilitation. It opens possibilities for neural analysis of different levels of conscious representation, as well as for studying coordination and integration of cerebral structures, providing verbal regulation and mediation of human conscious activity.

\section{9: Behavioural Exploration of Callosal Functioning in Dyslexic Children}

Maryse Lassonde ${ }^{1,3}$, Perchet Caroline ${ }^{2,3}$, Karyne Lavoie ${ }^{1,3}$ and Renee Beland ${ }^{1,3}$

1. Psychology, University of Montreal, Montreal, QC, Canada

2. INSERME342, Hopital Neurologique, Bron, France

3. Centre de Recherche, Hopital Ste-Justine, Montreal, QC, Canada

$\mathbf{R}$ eading is a higher-order mental activity that involves the participation of both hemispheres. There is conflicting evidence in the literature as to whether or not information flow between the hemispheres is deficient in dyslexic people due to a dysfunctional corpus callosum. To address this issue, we used a tactile localisation task and a visual letter discrimination task to investigate interhemispheric transfer of somatosensory, visuoperceptual and lexical information in 16 dyslexic children aged 8 to 13 years and 12 controls. In the tactile localisation task, which required intermanual transfer of a somatosensory stimulation applied to one hand, the performance of the dyslexic children was comparable to that of the controls. In the letter discrimination task, where they had to make same-different judgements of letters presented in one or both visual hemifields, the dyslexic children did not differ from the controls when the letters had to be compared on the basis of their physical characteristics. However, they obtained a significantly higher number of non-responses than the controls when the comparisons were based on lexical characteristics of the stimuli. This was true both for intrahemispheric and interhemispheric presentations. The results do not support the hypothesis of callosal dysfunction in dyslexic children. They rather suggest that dyslexic children are slower in accessing lexical information, most likely because they make insufficient use of phonological strategies in the encoding of written language.

\section{6: The Impact of Speech Impairment upon Subjective Identity Construction During Treatment}

Linda A. Petcopoulos ${ }^{1}$ and Jacinta Douglas ${ }^{2}$

1. Speech Pathology, Belmont Community Rehabilitation Centre, Jan Juc, VIC, Australia

2. Speech Pathology, LaTrobe University, Melbourne, VIC, Australia

The present study was designed to evaluate the impact of speech impairment upon one man's subjective identity construction before, during and after an intensive treatment program. The intensive treatment program 
was designed to improve speech intelligibility by encouraging and developing the participant's self-monitoring skills. The study involved two main aims. The first aim was to evaluate the effectiveness of an intensive treatment program upon speech intelligibility for a man with severe-chronic mixed spastic-ataxic dysarthria. The second was to develop an insight into the participant's current psychosocial level of functioning by monitoring impairment, subjective identity construction and social participation before, during and after an intensive treatment program. Subjective identity construction was viewed as a dynamic process through social interaction. The treatment evaluation for speech intelligibility employed a quantitaive method using single case time series analysis. Subjective identity construction was analysed through a qualitative method involving thematic analysis of in-depth interview transcripts. The findings revealed a significant increase in speech intelligibility scores at post-test and follow-up. They also revealed a reconstruction in subjective identity occurred for the participant over the length of the treatment program. It was concluded that the nature of the intensive treatment and the focus on his self-monitoring skills improved his speech intelligibility. It was also concluded that speech impairment impacted negatively upon the participant's identity at four years post-onset and that this changed over the length of the treatment program.

\title{
73093: The Role Of The Left Insula For Language Function
}

\author{
Mika Otsuki', Yoshiaki Soma², Yoshitsugu Nakagawa ${ }^{3}$, Akihisa Matsumoto $^{4}$ \\ and Hiroaki Naritomi ${ }^{5}$ \\ 1. School of Psychological Science, Health Sciences University of Hokkaido, Sapporo, Japan \\ 2. Soma Neurology Clinic, Niigata, Japan \\ 3. School of Nursing and Social Services, Health Sciences University of Hokkaido, Tobetsu, Japan \\ 4. Department of Neurology, Sapporo City General Hospital, Sapporo, Japan \\ 5. Department of Cerebrovascular Medicine, National Cardiovascular Center, Suita, Japan
}

Background and Purpose: Little is known about the role of the left insula cortex regarding language function. Several reports suggest that the area is related to articulation and auditory recognition. We examined the language function of 9 patients who had infarction in the left insula, Broca's area or both to clarify the role of the left insula for language function.

Methods: 9 right-handed Japanese patients (6 males, 3 females) who had language impairment after infarction were examined. Their lesions were assessed by MRI, and we administered the Western Aphasia Battery first within a month and followed it up to for several months to years.

Results: All the patients did not show articulatory impairment. All the patients showed difficulty in comprehension of sentences but not words. The patients whose lesions were involved the left middle frontal lobe showed comprehension impairment of words. The patients whose lesions were restricted to Broca's area demonstrated difficulty in word generation, but it was not severe and improved in several months. The patients who had lesions in the left anterior insula showed severe disability in word generation, while, those who had lesions in the left posterior insula showed only trivial difficulty.

Conclusion: (1) The left insula and Broca's area are not related to articulatory impairment. (2) Word comprehension impairment is found only when the lesions include the left middle frontal lobe. (3) Both Broca's area and 
the left insula cause difficulty in word generation, and the involvement of the left anterior insula makes the symptom severe and persistent.

\section{1: Neural Mechanism for Selective Impairment of Reading Following Occipital Lobe Damage}

Shimako Nishiyama', Mie Matsui ${ }^{2}$, Shigeki Taniguchi ${ }^{3,1}$ and Madoka Kurachi ${ }^{1}$

1. Inami General Hospital, Higashitonamigun, Japan

2. Department of Psychology, School of Medicine, Toyama Medical and Pharmaceutical University, Toyama, Japan

3. School of Medicine, Toyama Medical and Pharmaceutical University, Toyama, Japan

$\mathrm{T}$ The purpose of this study was to examine the neural mechanism of the selective impairment of reading. We evaluated two patients with left occipital lobe lesions in different locations. Patient 1 (with pure alexia, associative visual agnosia and right homonymous hemianopia) had a lesion in the left medial occipital lobe and the splenium of the corpus callosum on MRI. His Full Scale IQ was 67 (verbal IQ = 79, performance IQ = 57) and he showed pure alexia without aphasia on assessment with the Western Aphasia Battery (WAB). Patient 2 (with right homonymous hemianopia) showed a lesion in the left lateral occipital lobe on MRI. Her Full Scale IQ was 101 (verbal IQ $=101$, performance IQ $=83$ ) and had no aphasia on assessment with the WAB. We examined the ability to read 46 kanji (single ideograms), 46 kana and 26 alphabet letters (single phonograms) to investigate whether phonograms and ideograms are dissociated in oral reading. Patient 1 demonstrated a reading score for kanji that was significantly higher than that for kana $(p<.01)$ or that for alphabet letters $(p<.01)$. The scores for kana were not significantly different from those for alphabet letters. Moreover, there were no significant differences between the scores for high complexity kanji ( $>6$ strokes) and low complexity kanji ( $<6$ strokes). Patient 2 showed a perfect score on each task. This study revealed that a lesion in the left medial occipital lobe and the splenium of the corpus callosum can impair the ability to read phonograms independently of the complexity of the letters. We suggest that the process of phonogram reading is mediated by these regions.

\section{2: Semantic Knowledge Impairment in Patients with Unilateral Temporal Lobe Damage: Effect of Aetiology of Lesion on Access and Storage}

Michelle A. Robison ${ }^{1}$, Natalie A. Lambert ${ }^{1}$, Diana Caine ${ }^{1}$ and Laurie A. Miller ${ }^{2}$

1. School of Psychology, University of Sydney, Sydney, NSW, Australia

2. Neuropsychology Unit, Royal Prince Alfred Hospital, Sydney, NSW, Australia

Tn a study of patients with temporal lobe lesions of different etiologies we Ifound the right temporal (RT) group was impaired at recognising photographs of famous faces, but not of famous buildings, and the left temporal (LT) group was poor at naming people, buildings and everyday objects from pictures and description. In the present investigation, we explored whether aetiology affects the ability to access knowledge from these semantic categories on the basis of pictures versus descriptions. Patients with LT (2 lobectomy, 4 stroke, 2 focal atrophy) and RT (3 lobectomy, 5 stroke, 1 atrophy) lesions were compared to 12 normal control subjects. Patients with temporal lobe atrophy of either hemisphere (LTA and RTA) demonstrated near-pervasive deficits in recalling names and specific semantic details 
about people, buildings and objects from both pictures and description as well as in recognising which face or building was famous. LT lobectomy (LTL) patients had difficulty naming people, buildings and objects, largely irrespective of whether presented with pictures or definitions, whereas LT stroke patients were only impaired at naming pictures of buildings. The LTL and LTA patients were also deficient at recognising which object name fit a description. RT lobectomy and RT stroke caused no impairments. Hence, aetiology of temporal lesion had a significant impact on pattern of semantic knowledge deficit, with focal atrophy causing the most dramatic impairments. Given that the temporal pole was spared in the stroke cases, it is argued that the LT anterior neocortex is a repository for knowledge about common objects.

\section{4: The Effect of Unilateral Temporal Lobe Lesions on Face Recognition and Access To Person-specific Knowledge}

Natalie A. Lambert ${ }^{1}$, Michelle A. Robison ${ }^{1}$, Laurie A. Miller ${ }^{2}$ and Diana Caine ${ }^{1}$

1. School of Psychology, University of Sydney, Sydney, NSW, Australia

2. Neuropsychology Unit, Royal Prince Alfred Hospital, Sydney, NSW, Australia

The roles of the right and left temporal lobes in the processing of faces and person-specific knowledge are still to be clearly defined. Does access to person knowledge differ from other kinds of objects with respect to laterality of lesion effects? Are laterality effects mediated by stimulus modality (i.e., pictures versus descriptions)? This study aimed to investigate these questions by comparing patients with left temporal (LT) $(n=8)$ and right temporal (RT) lesions $(n=11)$ to normal control subjects $(n=12)$ on verbal and visual tests of people, buildings and objects. The RT group was impaired at recognising which was a famous face, but not at choosing pictures of famous buildings or famous names from non-famous distractors. The LT group was impaired at naming people, buildings and objects, when presented with either a picture or a verbal description of the item. If face recognition per se is controlled for, there was no difference between right and left temporal patients in producing person-specific knowledge to faces or names. Thus the right temporal lobe seems to be important for the perceptual processing of faces (face recognition per se) but not the visual recognition of other kinds of objects. The left temporal lobe is important for naming, irrespective of category, or stimulus modality. There was no evidence from these results that the RT lobe differs from the left in accessing information about people across modalities.

\section{5: Impairment of Visual Knowledge and Imagery of Others' Belongings: A Case Study of Partial Disturbance of "Categorical Attitude"}

Kozue Saito ${ }^{1}$, Mika Otsuki², Kazuyuki Nagatsuka ${ }^{1}$ and Hiroaki Naritomi ${ }^{1}$

1. Cerebrovascular Division, Department of Medicine, National Cardiovascular Centre, Osaka, Japan

2. School of Psychological Science, Health Sciences University of Hokkaido, Hokkaido, Japan

$\mathrm{W}$ e report a patient who suffered impairment of visual knowledge and imagery of other people's belongings. The patient was 74-year-old right-handed Japanese woman who had an infarction in the left temporooccipital lobe and hippocampus. Neurological examinations revealed that she had right upper quadrantanopia but no motor or sensory impairment. General intelligence was preserved, and her memory disturbance was not significant. 
Formal language examinations demonstrated that she had two-way anomia, pure alexia and agraphia of Kanji (Japanese morphograms). Prosopagnosia and topographical amnesia were not present. Detailed examinations revealed her characteristic impairment. First, she had difficulty in confrontation naming, but she was able to name from tactile and auditory information and name by word definition. However, this symptom was not attributed only to visual agnosia because she was not able to spontaneously draw pictures nor retrieve any visual information (shape, colour and so on) of the objects, although she was able to copy the drawings well. There was only one exceptional category: her own belongings. She visually recognised all her belongings and retrieved their visual information. For example, when the photo of her wardrobe was presented, she immediately named it "wardrobe", while when the examiner's wardrobe was presented, she hesitatingly named it "hatch". Goldstein described the possibility of disturbance of "categorical attitude" (abstract attitude) as one of the mechanisms of anomia (1948). We concluded that presumably the patient had the disturbance of partial "categorical attitude" limited to visual knowledge and imagery.

\section{4: The Relatedness of Drawing Quality and Naming in Aphasia}

\section{Dana Farias and Christine Davis}

Speech Pathology, University of California @ Davis, Medical Centre, Sacramento, CA, USA

$\mathrm{P}$ revious research with aphasics has demonstrated a conceptual link between drawing and language such that drawing reflects the integrity of the semantic-lexical system. Our current research suggested that drawing can be used to access the semantic-lexical system thereby facilitating naming. The purpose of this study was to determine the relationship between drawing quality, naming, handedness of drawing and classification and severity of aphasia. We proposed the following hypotheses: (1) more detailed drawings would be related to higher naming scores by systematically accessing the perceptual features of the object which would activate the semantic-lexical network to a threshold sufficient for accurate word selection and production; (2) higher word-picture match scores would be related to drawings that were rated higher on quality and detail reflecting the integrity of the semantic-lexical system; (3) drawing quality would be unrelated to severity or classification of aphasia. Twenty-two aphasic patients were administered a modified version of the Reading Comprehension Battery for Aphasia. Scores on confrontational naming, naming while drawing and picture-word match were obtained. Drawings were analysed by multiple-raters to assess the quality and detail of their drawings. Results supported previous findings that severity and classification of aphasia were unrelated to drawing quality. Contrary to our predictions drawing quality did not correlate to semantic-lexical measures, nor was quality related to naming abilities. However, there was a significant correlation of handedness to naming with dominant handedness producing more accurate naming, but not better drawing quality. The role of drawing in a language model is discussed. 


\section{4: Neuropsychological Sequelae of Exposure to Toxic Anhydrous Ammonia}

David M. Mahalick ${ }^{1,2}$, John P. Greenberg ${ }^{4}$ and John McGinley ${ }^{1}$

1. Neurosurgery \& Pediatrics, NJ Med School, Newark, NJ, USA

2. Pediatrics, Robert Wood Johnson Med School, New Brunswick, NJ, USA

3. Applied Psychology, New York University, New York, NY, USA

4. Neurology, Robert Wood Johnson Med School, New Brunswick, NJ, USA

Objective: Currently, there is no literature that describes the neurological, and neuropsychological sequelae of toxic exposure to anhydrous ammonia. The purpose of this study is to outline the symptom complex secondary to significant exposure to anhydrous ammonia.

Method: The study group consisted of 17 males between the ages of 27 and 57. Subjects were physician referred to rule out suspected neurological/neuropsychological deficit secondary to toxic exposure. The group was divided into those subjects who sustained "High Exposure" versus "Moderate Exposure". Subjects underwent Neuropsychological and Neurological Examination (including EMG). Performances of the "High and Moderate" exposure groups were compared on various neuropsychological and neurological examinations. Performances were also compared to the general "normal" population. Various a priori assumptions were established regarding performances between the two study groups and the normal population.

Results: Preliminary statistical analyses utilising $t$ tests demonstrated significant differences between clinical and normal populations on neuropsychological functioning, usually at levels less than $p<.05$. Most subjects in this study demonstrated abnormalities on neurological examination as well as on EMG studies. Comparisons between "High Exposure" and "Moderate Exposure" groups demonstrated performances in the predicted direction, with T-tests usually significant far below the $p<.05$ level.

Conclusions: Given the known effects of hyperammonaemia on brain functioning resulting from other etiologies, our a priori predictions (viz., that patients who were exposed to toxic anhydrous ammonia would demonstrate neurological and neuropsychological dysfunction) were confirmed upon statistical analyses. Moreover, those with high levels of exposure performed more poorly than those with only moderate levels.

\section{6: Mitochondrial Encephalomyopathy, Lactic Acidosis and Stroke-like Episodes: A Case Study}

Joanne Oram and Helen Tinson

Neurosciences, Princess Alexandra Hospital, Wooloongabba, QLD, Australia

itochondria are organelles that are scattered throughout the cytoplasm
of cells. They contain enzymes that are necessary for cellular respira-
tion, and represent the energy source of the cells throughout the body. There
are a broad range of diseases that have in common, a dysfunction of mito-
chondrial metabolism. Multiple organs and systems of the body may be
affected, including the central nervous system, resulting in a very diverse
clinical picture. Mitochondrial Encephalomyopathy, Lactic Acidosis and
Stroke-Like Episodes (MELAS) is a variant with symptoms that include:
poor growth, focal or generalised seizures, recurrent acute episodes that
resemble strokes or prolonged ischemic attacks, migraine-like headaches,
vomiting and episodic lactic acidosis. Some studies of the neuropsycholog-
ical effects of MELAS show a generalised cognitive deterioration. Others 
have shown a distinct pattern of impairment including difficulties in language, perception, attention and executive skills, and sometimes memory. This study describes a 28-year-old male, referred for neuropsychological assessment following an ischemic event and subsequent diagnosis of MELAS. Neuropsychological assessment indicated strength in the areas of language, verbal memory and verbal reasoning. Weakness was identified in the areas of attention, working memory/executive skills, speed of processing and the ability to process, organise and recall visuospatial information. Evidence for a pattern of specific cognitive deficits, rather than a global dementia is discussed, along with the functional implications of the identified cognitive deficits.

\title{
73118: Latency, Accuracy and Meta-Cognition of HIV Positive Individuals in the Performance of Complex Tasks
}

\author{
Kenneth L. Rider ${ }^{1}$, David J. Hardy ${ }^{2}$, Steven A. Castellon ${ }^{2,3}$, Robert Schug ${ }^{2}$ \\ and Charles H. Hinkin ${ }^{2,3}$ \\ 1. Dept. of Psychiatry and Behavioral Sciences, Stanford University School of Medicine, Stanford, \\ CA, USA \\ 2. Dept. of Psychiatry and Biobehavioral Sciences, UCLA School of Medicine, Los Angeles, CA, USA \\ 3. VA Greater Los Angeles Healthcare System, Los Angeles, CA, USA
}

Drior research on simple reaction-time tasks suggests that HIV positive individuals perform more slowly than HIV negative individuals. We were interested in whether this effect occurred on more complex tasks and in potential reasons why latency and accuracy might differ between these individuals. We administered items 4 through 19 of the WAIS-III Matrix Reasoning test to 47 HIV positive and 50 HIV negative participants and recorded response, response latency, and confidence on a 4-item Likert scale for each item. For each group of participants, we calculated average accuracy and average log latency on each item, prepared Brinley charts and conducted a series of regression analyses using items as the data points. We found that both groups took similar times to answer each item correctly, with latency increasing as item complexity increased. However, the HIV positive group tended to make proportionately more errors (about 33\%) on each item. This result is in contrast to performance on simple reaction time tasks where both groups tend to be equally accurate, but where HIV positive individuals tend to be slower. Further analysis comparing items answered correctly to item answered incorrectly for each group suggested that HIV positive individuals were sacrificing accuracy for speed. We also found that compared with negative individuals, positive individuals tended to be unrealistically optimistic about the accuracy of responses they rated as "quite sure" and unrealistically pessimistic about responses rated as "just a guess". These results suggest a less effective metacognition on the part of HIV positive individuals.

\section{6: Neuropsychological Problems Associated with Chronic Fatigue Syndrome: A Meta-analytic Review}

Susan J. Cockshell and Jane Mathias

Psychology, The University of Adelaide, Adelaide, SA, Australia

Chronic fatigue syndrome (CFS) is characterised by long-standing excessive fatigue, which cannot be explained by known medical or psychi- 
atric conditions, is not substantially alleviated by rest, and is disproportionate to the level of exertion. CFS is often also associated with subjective reports of cognitive problems. Neuropsychological investigations of CFS have reported deficits in the areas of memory, learning, and information processing speed, with normal levels of intellectual and executive functioning. However, the research findings are inconsistent and have not been adequately consolidated, thereby limiting the extent to which they inform clinical practice. The current study therefore provides a meta-analysis of research conducted between 1988 and 2004, which examined the neuropsychological consequences of CFS. A comprehensive search of the psychological and medical literature databases and 21 relevant journals was undertaken, using 9 search terms. All identified articles were then screened using detailed inclusion and exclusion criteria. Effects sizes, percentage overlap between CFS and healthy control groups, and failsafe Ns (i.e., number of studies with non-significant findings required in order to reverse a significant result) were calculated for these studies in order to evaluate this research independently of the effects of sample size (which influences statistical power) and the bias introduced by a tendency to publish significant results. These statistics enabled a direct comparison of the findings derived from different tests of the same cognitive ability (e.g., memory) and from different cognitive functions, in order to identify the tests and cognitive abilities that best discriminate between groups.

\title{
73175: Carbon Monoxide Poisoning as a Suicide Attempt: 12-month Neuropsychological and Psychiatric Outcomes
}

\author{
Phillipa J. Hay ${ }^{1}$ and Linley A. Denson ${ }^{2,3}$ \\ 1. School of Medicine, James Cook University, Townsville, QLD, Australia \\ 2. Psychology Department, University of Adelaide, Adelaide, SA, Australia \\ 3. Clinical Psychology Department, Royal Adelaide Hospital, Adelaide, SA, Australia
}

Aims: To prospectively evaluate neuropsychological and psychiatric outcomes following carbon monoxide (CO) poisoning. A consecutive series of 41 adults, with significant $\mathrm{CO}$ exposure as a suicide attempt, underwent neuropsychological and psychiatric assessment within a mean of 3.1 days of hospital admission. They were compared with matched controls, presenting with a non-neurotoxic suicide attempt. The most common psychiatric diagnoses were major depression and adjustment disorder with depressed mood. Many had alcohol abuse or dependence. Initially controls and $\mathrm{CO}$ subjects showed similar cognitive impairment (except for $4 \mathrm{CO}$ subjects with very severe impairment), but controls were more depressed. At 2 months, trends were generally towards improvement in all participants, with no between-group differences (reported in 1). Some CO subjects had brain MRI. Twenty-eight $(68 \%)$ of the CO group were reviewed at 1 year, with further improvement in cognitive functioning, depressive symptoms and general functioning (median Axis V GAF scores 76.5). Three had made another suicide attempt. One had completed suicide. One neuropsychological performance (Rey AVLT delayed recall) predicted outcome on the GAF at 12 months.

Conclusions: Except where CO toxic effects were severe, most psychosocial dysfunction at follow-up appeared due to psychiatric disorder. The study did not support $\mathrm{CO}$ exposure exacerbating mood disorder in this sample. 


\title{
73177: Relationships between Quality of Life, Cognitive and Emotional Function in Survivors of Acute Respiratory Distress Syndrome
}

\author{
Ramona O. Hopkins ${ }^{1,2}$, Lindell K. Weaver ${ }^{2}$, Karen J. Chan ${ }^{3}$ and James F. Orme ${ }^{2}$ \\ 1. Psychology Department and Neuroscience Center, Brigham Young University, Provo, UT, USA \\ 2. Pulmonary and Critical Care Medicine, LDS Hospital, SLC, UT; 3. Statistical Data Center, LDS Hospital, \\ $S L C, U T, U S A$
}

Background: Acute Respiratory Distress Syndrome (ARDS) affects more than 150,000 people per year in the United States. ARDS is characterised by lung injury and hypoxemia, and has a mortality rate of $11 \%$ to $43 \%$. Previous research indicates ARDS survivors have cognitive impairments, depression, and anxiety. However there is no information in the literature regarding which of these factors (i.e., cognitive, depression, or anxiety) are associated with decreased quality of life. The purpose of the study was to assess the relationships between quality of life and cognitive and emotional function in ARDS survivors, 1-year post-hospital discharge.

Methods: Sixty-six ARDS survivors were administered a battery of neuropsychological tests, measures of affect (BDI and BAI), and quality of life (SF-36) 1-year post-hospital discharge. Patient demographic and medical data (length of stay, laboratory values, and ventilator data) were recorded. Cognitive impairment was defined as scores on 2 or more neuropsychological tests that were $>1.5 S D$ below the normative population mean. Results: Of 66 ARDS patients, $55 \%$ were female, with a mean $\pm S D$ : age $=46 \pm 16.4$ years, mean education level $=13 \pm 2.2$ years, APACHE II score $=17.9 \pm 6.2$, hospital length of stay $=39.1 \pm 21.5$ days, $\mathrm{PaO} 2 / \mathrm{FiO} 2$ ratio $=104.5 \pm 32$ Torr. Forty-five percent of the patients had cognitive sequelae; $28 \%$, mild to moderate symptoms of depression; and $28 \%$, mild to moderate symptoms of anxiety. Decreased quality of life was significantly correlated with increased depression and anxiety ( $p=.000$ to .003$)$. Decreased quality of life was significantly correlated with decreased intellectual function (FSIQ; $p=.01$ ).

Discussion: ARDS survivors experience cognitive impairments, depression and anxiety, that are associated with decreased quality of life 1 year posthospital discharge.

\section{0: Neurological, Psychiatric and Neuropsychological Sequelae over a Three Year Period for a Case of a Rare Multi-system Degenerative Neurological Disorder: Neuroacanthocytosis}

\author{
Amy A. Scholes and Catherine E. Meade \\ Neuropsychiatry; Adult Psychiatry and CAMHS, Royal Melbourne and Alfred Hosptials, Melbourne, \\ VIC, Australia
}

Jeuroacanthocytosis (NA) is a rare multi-system degenerative neurolog-
ical condition characterised by psychiatric symptoms alongside neuro-
logical signs and acanthocytosis in peripheral blood (i.e., spiked red blood
cells) and normal lipoproteins. Diagnosis is complicated by the variable
array of NA syndromes, the rarity of the disorder, and the way the symp-
toms manifest. Initially Learning Disorder, Tourettes Syndrome, OCD and
epilepsy are amongst the typical diagnoses made. A case is presented of this
rare disorder, demonstrating the psychiatric, neurological and neuropsycho-
logical sequelae over a longitudinal (three year) period. This patient (KT)
had a history of OCD features, learning difficulty, and tics, which initially
led to a tentative diagnosis of Tourettes syndrome. Epilepsy then emerged 
and it was when he was being assessed for epilepsy surgery that the diagnosis of NA was finally made. He underwent thorough assessment at this time, then again three years later subsequent to a decline in behaviour and physical symptoms, which were causing significant management problems for his family. Neuropsychologically a pattern of ongoing memory impairment, executive dysfunction, behavioural/personality change and reduced insight were evidenced. In regard to his neurological symptoms, his tongue and lip biting, and his feeding dystonia were causing increasing problems over time. MRI scan showed severe bilateral atrophy of the head of the caudate nucleus and some prominence of the frontal horns of the left ventricle. Psychiatrically, he was showing symptoms of a depressed mood, irritability, anger outbursts, as well as a period of shoplifting. Notably his OCD symptoms had resolved over the three year period. This represents another case to add to the limited literature around this rare disorder.

\title{
73234: Attentional Functions Following Different Neurotoxic Exposures
}

\author{
Tatjana Novakovic-Agopian', Sabine Gysens ${ }^{2,1}$ and Rosemarie M. Bowler ${ }^{2}$ \\ 1. California Pacific Medical Center, San Francisco, CA \\ 2. San Francisco State University, San Francisco, CA, USA
}

\begin{abstract}
$\mathrm{O}^{-1}$ ne of the most common sequelae of neurotoxic exposure involves difficulties in the areas of attention and speed of processing. We compared three groups of neurotoxically exposed workers and a group of 194 control subjects with a computerised Visual Attention Test (VAT) battery. The exposed groups included 15 individuals exposed to nerve gas (sarin and mustard gas), 176 hazardous waste workers exposed to ethylene dichloride (EDC), and 40 welders exposed to manganese fumes. The VAT battery includes computerised attentional tests that assess speed of processing information, ability to orient and covertly shift attention, and ability to inhibit automatic orienting. These tests are based on the cognitive neuroscience and neuroimaging attentional research. All exposed groups had slower reaction time (RT) relative to controls. This finding was significant for welders and EDC exposed workers; a trend was observed for nerve gas exposed workers. Whereas EDC exposed workers and welders had more difficulty inhibiting automatic orienting than control subjects, this was not true for individuals exposed to nerve gas, who showed the same pattern as controls. The nerve gas exposed group had a disproportionally large slowing of RT at brief interval durations for the inhibition of automatic orienting task as compared to controls and other exposed groups. Both EDC exposed workers and welders had more difficulty on the automatic orienting task then controls and individuals exposed to nerve gas. They had a larger validity effect; their RT was disproportionally slower when their attention was misguided to a location different from the one where the target appeared. While both controls and nerve gas exposed individuals showed a normal pattern of inhibition of return at longer interval durations, both EDC exposed workers and welders did not show this pattern. On longer cue-totarget interval duration $(800 \mathrm{~ms})$, they continued to have longer RT on invalid trials than on valid ones.
\end{abstract}

